\title{
A!
}

This is an electronic reprint of the original article.

This reprint may differ from the original in pagination and typographic detail.

Chen, Hongyang; Ahmad, Fauzia; Vorobyov, Sergiy; Porikli, Fatih

\section{Tensor decompositions in wireless communications and mimo radar}

Published in:

IEEE Journal on Selected Topics in Signal Processing

DOI:

10.1109/JSTSP.2021.3061937

Published: 01/04/2021

Document Version

Peer reviewed version

Please cite the original version:

Chen, H., Ahmad, F., Vorobyov, S., \& Porikli, F. (2021). Tensor decompositions in wireless communications and mimo radar. IEEE Journal on Selected Topics in Signal Processing, 15(3), 438-453. [9362250].

https://doi.org/10.1109/JSTSP.2021.3061937

This material is protected by copyright and other intellectual property rights, and duplication or sale of all or part of any of the repository collections is not permitted, except that material may be duplicated by you for your research use or educational purposes in electronic or print form. You must obtain permission for any other use. Electronic or print copies may not be offered, whether for sale or otherwise to anyone who is not an authorised user. 


\title{
An Overview of Tensor Decompositions in Wireless Communications and MIMO Radar
}

\author{
Hongyang Chen, Senior Member, IEEE, Fauzia Ahmad, Fellow, IEEE, Sergiy Vorobyov, Fellow, IEEE, \\ and Fatih Porikli, Fellow, IEEE,
}

\begin{abstract}
The emergence of big data and the multidimensional nature of wireless communication signals present significant opportunities for exploiting the versatility of tensor decompositions in associated data analysis and signal processing. The uniqueness of tensor decompositions, unlike matrix-based methods, can be guaranteed under very mild and natural conditions. Harnessing the power of multilinear algebra through tensor analysis in wireless signal processing, channel modeling, and parametric channel estimation provides greater flexibility in the choice of constraints on data properties and permits extraction of more general latent data components than matrix-based methods. Tensor analysis has also found applications in Multiple-Input Multiple-Output (MIMO) radar because of its ability to exploit the inherent higher-dimensional signal structures therein. In this paper, we provide a broad overview of tensor analysis in wireless communications and MIMO radar. More specifically, we cover topics including basic tensor operations, common tensor decompositions via canonical polyadic and Tucker factorization models, wireless communications applications ranging from blind symbol recovery to channel parameter estimation, and transmit beamspace design and target parameter estimation in MIMO radar.
\end{abstract}

Index Terms-Tensor decomposition, tensor factorization, rank, parallel factor analysis (PARAFAC), Tucker model, CDMA, MIMO, symbol recovery, millimeter wave, transmit beamspace, radar.

\section{INTRODUCTION}

A tensor is a multidimensional array. A first-order tensor is a vector, a second-order tensor is a matrix, and tensors of order three or higher are generalized matrices called higherorder tensors. An $N$ th-order tensor is an element of the tensor product of $N$ vector spaces [1]-[5]. Tensor algebra is generalized from matrix algebra, thus they have many similarities but they also have different properties. Higher-order tensors and their decompositions have recently become pervasive in signal, data analytics and machine learning techniques.

The roots of multiway data analysis can be traced back to studies of homogeneous polynomials by Hitchcock in the late 1920s [6], [7], followed by other contributions including those by Tucker [8]-[10], Carroll and Chang [11], and Harshman [12]. The Tucker decomposition (TKD) for tensors was introduced in psychometrics [9], [10], while the canonical

H. Chen is with the Research Center for Intelligent Network, Zhejiang Lab, Hangzhou 311121, China. (e-mail: dr.h.chen@ieee.org).

F. Ahmad is with Temple University, Philadelphia, PA 19085, USA. e-mail: fauzia.ahmad@temple.edu)

S. Vorobyov is with Aalto University, Finland. e-mail: (svor@ieee.org, sergiy.vorobyov@aalto.fi)

F. Porikli is with Australian National University, Australia. e-mail: (fatih.porikli@anu.edu.au) polyadic decomposition (CPD) was independently discovered and put in an application context under the names of canonical decomposition (CANDECOMP) in psychometrics [11] and parallel factor model (PARAFAC) in linguistics [12]. Besides the developments in psychometrics, tensor decompositions have been examined and applied in other fields, such as chemometrics, the food industry, social sciences [13], [14], and signal processing [15]-[17].

With regard to signal processing in wireless communications, the received signal is multidimensional in nature and may exhibit a multilinear algebraic structure [18]. However, owing to the broad system variety with differing yet complex transmission structures, realistic channel models, and efficient receiver signal processing, wireless communications offer new challenges for applying tensor decompositions. A high-speed wireless transmission is impacted by various factors in the physical layer, such as interference from different sources, attenuation of signal power with distance, and other signal fading effects of the wireless communication channel. At the receiver, signal processing is generally used to combat multipath fading effects, inter-symbol interference (ISI), and multiuser (co-channel) interference by means of multiple receive antennas. Wireless communication systems employing multiple antennas at both ends of the link, commonly known as Multiple-Input Multiple-Output (MIMO) systems, are being considered as one of the key technologies to be deployed in current and upcoming wireless communications standards [19]. Generalized tensor decompositions are typically required to cover the disparate communication system types. Besides, tensor decompositions can also be used to address the sensor array processing problem, such as the blind spatial signature [24]. The tensor approach can loose many restrictive assumptions which are required by many conventional approaches.

In [20]-[22], the authors examined the integration of multiple-antenna and Code-Division Multiple-Access (CDMA) technologies. As described in [25], [26], [28], tensor modeling based MIMO systems have been demonstrated to potentially provide high spectral efficiencies by capitalizing on spatial and code multiplexing. Furthermore, for a thirdorder received signal tensor, each signal sample is an element of a three-dimensional (3-D) tensor and is represented by three indices, each associated with a specific type of systematic variation of the received signal. In such 3-D space, each dimension of the received signal tensor can be interpreted as a particular form of signal "diversity". In most cases, two of the three dimensions account for space and time. The third dimension, however, depends on the specific wireless commu- 
nication system considered. For instance, the third dimension can correspond to frequency in MIMO Orthogonal Frequency Division Multiplexing (MIMO-OFDM) system [29]. By means of Space-Time-Frequency (STF) coding [30]-[33], the MIMOOFDM communication systems are able to achieve high data rates and combat fading effects [34], [36]-[40]. In [41][43], [82]-[85], the authors investigated cooperative relayassisted MIMO communications, which have emerged as a popular means for enhanced wireless system performance, improved quality of service, and cost and structure reduction. Tensor-based approaches have gained considerable attention in cooperative MIMO communication systems.

MIMO radar technology has garnered substantial research interest over the last decade and has found applications in over-the-horizon radar, maritime radar, automotive radar, and dual-function radar-communications, to name a few [44]-[55]. A MIMO radar with multiple colocated transmit and receive antennas can estimate target parameters of interest through simultaneous transmission of several orthogonal waveforms and coherent processing of the radar returns. Although the antennas constituting the transmit array or the receive array are closely spaced, the arrays themselves may not be colocated, as is the case in bistatic MIMO radar. The configuration with colocated transmit and receive arrays, on the other hand, is called a monostatic MIMO radar. Proper exploitation of waveform diversity and degrees-of-freedom offered by the multi-antenna transmit/receive configurations for interference suppression and resolution enhancement can lead to improvements in target detection and parameter estimation performance over a conventional radar. Similar to wireless communications, MIMO radar signal processing can benefit from tensor analysis in successfully achieving reliable and effective target parameter estimation [56]-[60].

The main purpose of this paper is to provide a comprehensive overview of tensor decompositions in the application areas of wireless communications and MIMO radar. Towards this objective, in Section II, we review some basic tensor operations and common tensor decompositions, including Tucker and CPD. The uniqueness of the decompositions is also briefly discussed. Section III provides a detailed survey of tensor analysis in wireless communications, ranging from blind symbol recovery to time-varying channel modeling and parameter estimation for different systems, including multiuser CDMA, cooperative/relay systems, and millimeter wave (mmWave) communication systems. In Section IV, we present an overview of tensor-based methods in MIMO radar, focusing on target localization and transmit beamspace (TB) design. Section V provides conclusions. It is noted that the topics and related research that we have showcased in this paper are by no means exhaustive. Rather, they inform the reader about the type of opportunities present in the considered application areas for employing tensor algebra and decompositions.

Notation: Scalars, column vectors, matrices, and tensors are denoted by lowercase, boldface lowercase, boldface uppercase, and calligraphic uppercase letters, such as $a, \mathbf{a}, \mathbf{A}$ and $\mathcal{A}$, respectively. The vector $\mathbf{a}_{i}$ (resp. $\mathbf{a}_{j}$ ) represents the $i$ th row (resp. $j$ th column) of matrix $\mathbf{A}$. The operations $\mathbf{A}^{T}, \mathbf{A}^{*}$, $\mathbf{A}^{H}, \mathbf{A}^{-1}$, and $r_{\mathbf{A}}$ denote the transpose, the conjugate, the conjugate (Hermitian) transpose, the Moore-Penrose pseudoinverse, and the rank of $\mathbf{A}$, respectively. The operator $D_{i}(\cdot)$ forms a diagonal matrix from the elements of the $i$ th row of its matrix argument. The symbols $\circ, \otimes$, and $\diamond$ represent outer product, Kronecker product, and Khatri-Rao product, respectively. The remaining notation should be clear from the context.

\section{BASIC TENSOR OPERATIONS AND DECOMPOSITIONS}

In this section, we review some useful matrix products, basic tensor operations, and common tensor decompositions [61][64]. These establish the preliminaries for the applicationspecific descriptions that follow in subsequent sections.

\section{A. Basic Definitions and Operations}

Definition 1. Kronecker product of two matrices: The Kronecker product of $\mathbf{A} \in \mathbb{C}^{I \times J}$ and $\mathbf{B} \in \mathbb{C}^{M \times N}$ is defined as

$$
\mathbf{A} \otimes \mathbf{B}=\left[\begin{array}{cccc}
a_{1,1} \mathbf{B} & a_{1,2} \mathbf{B} & \cdots & a_{1, J} \mathbf{B} \\
a_{2,1} \mathbf{B} & a_{2,2} \mathbf{B} & \cdots & a_{2, J} \mathbf{B} \\
\vdots & \vdots & \ddots & \vdots \\
a_{I, 1} \mathbf{B} & a_{I, 2} \mathbf{B} & \cdots & a_{I, J} \mathbf{B}
\end{array}\right] \in \mathbb{C}^{I M \times J N}
$$

Considering additional matrices $\mathbf{C} \in \mathbb{C}^{J \times P}, \mathbf{D} \in \mathbb{C}^{Q \times P}$, and $\mathbf{E} \in \mathbb{C}^{Q \times M}$, we have the following properties:

\section{Property 1.}

$$
\operatorname{vec}\left(\mathbf{A} \mathbf{C D}^{T}\right)=(\mathbf{D} \otimes \mathbf{A}) \operatorname{vec}(\mathbf{C}) \in \mathbb{C}^{I Q} .
$$

where $\operatorname{vec}(\cdot)$ denotes columnwise vectorization of its matrix argument.

\section{Property 2.}

$$
(\mathbf{A} \otimes \mathbf{E})(\mathbf{C} \otimes \mathbf{B})=(\mathbf{A C}) \otimes(\mathbf{E B}) \in \mathbb{C}^{I Q \times P N} .
$$

Definition 2. Khatri-Rao product of two matrices: The Khatri-Rao product of $\mathbf{A} \in \mathbb{C}^{M \times J}$ and $\mathbf{B} \in \mathbb{C}^{N \times J}$ is defined as the column-wise Kronecker product,

$$
\begin{aligned}
& \mathbf{A} \diamond \mathbf{B}= \\
& {\left[\mathbf{a}_{1} \otimes \mathbf{b}_{1} \mathbf{a}_{2} \otimes \mathbf{b}_{2} \cdots \mathbf{a}_{J} \otimes \mathbf{b}_{J}\right] \in \mathbb{C}^{M N \times J} .}
\end{aligned}
$$

The Khatri-Rao product $\mathbf{A} \diamond \mathbf{B}$ can also be calculated as

$$
\mathbf{A} \diamond \mathbf{B}=\left[\begin{array}{c}
\mathbf{B} D_{1}(\mathbf{A}) \\
\vdots \\
\mathbf{B} D_{I}(\mathbf{A})
\end{array}\right] .
$$

Definition 3. Inner product of two tensors: The inner product of two tensors $\mathcal{A} \in \mathbb{C}^{I_{1} \times \ldots \times I_{M}}$ and $\mathcal{B} \in \mathbb{C}^{I_{1} \times \ldots \times I_{M}}$ of the same order $M$ is defined as

$$
\langle\mathcal{A}, \mathcal{B}\rangle=\sum_{i_{1}=1}^{I_{1}} \sum_{i_{2}=1}^{I_{2}} \ldots \sum_{i_{M}=1}^{I_{M}} a_{i_{1}, i_{2}, \ldots, i_{M}} b_{i_{1}, i_{2}, \ldots, i_{M}} .
$$

Definition 4. Outer product of two tensors: The outer product of an $M$ th order tensor $\mathcal{A} \in \mathbb{C}^{I_{1} \times \ldots \times I_{M}}$ and an $N$ th order tensor $\mathcal{B} \in \mathbb{C}^{J_{1} \times \ldots \times J_{N}}$ is defined as the $(M+N)$ th order tensor $\mathcal{A} \circ \mathcal{B}$ with elements

$$
(\mathcal{A} \circ \mathcal{B})_{i_{1}, i_{2}, \ldots, i_{M}, j_{1}, j_{2}, \ldots, j_{N}}=a_{i_{1}, i_{2}, \ldots, i_{M}} b_{j_{1}, j_{2}, \ldots, j_{N}} .
$$


Definition 5. Mode- $n$ product of a tensor and a matrix: Consider a tensor $\mathcal{A} \in \mathbb{C}^{I_{1} \times \ldots \times I_{N}}$ and a matrix $\mathbf{X} \in$ $\mathbb{C}^{L \times I_{n}}$, with $I_{n}$ equal to the dimension of the $n$th mode of $\mathcal{A}$. The mode- $n$ product between the tensor $\mathcal{A}$ and the matrix $\mathbf{X}$ yields an $N$ th order tensor $\mathcal{B}=\mathcal{A} \times{ }_{n} \mathbf{X} \in$ $\mathbb{C}^{I_{1} \times \ldots \times I_{n-1} \times L \times I_{n+1} \times \ldots \times I_{N}}$ such that

$$
\begin{aligned}
& b_{i_{1}, \cdots, i_{n-1}, l, i_{n+1}, \cdots, i_{N}}= \\
& \sum_{i_{n}=1}^{I_{n}} a_{i_{1}, \cdots, i_{n-1}, i_{n}, i_{n+1}, \cdots, i_{N}} x_{l, i_{n}} .
\end{aligned}
$$

Definition 6. Rank-one tensor: The tensor $\mathcal{A} \in \mathbb{C}^{I_{1} \times \ldots \times I_{M}}$ is said to be a rank-one tensor if it can be expressed as the outer product of $M$ vectors $\mathbf{v}_{m} \in \mathbb{C}^{I_{m}}$, with $m \in[1, M]$ as

$$
\mathcal{A}=\mathbf{v}_{1} \circ \mathbf{v}_{2} \cdots \circ \mathbf{v}_{M} .
$$

The entries of $\mathcal{A}$ can be presented as $a_{i_{1}, i_{2}, \ldots, i_{M}}=$ $v_{1}^{\left(i_{1}\right)} \ldots v_{M}^{\left(i_{M}\right)}$. Fig. 1 illustrate a rank-one tensor of order 3 , represented as the outer product of vectors $\mathbf{a}, \mathbf{b}$, and $\mathbf{c}$.
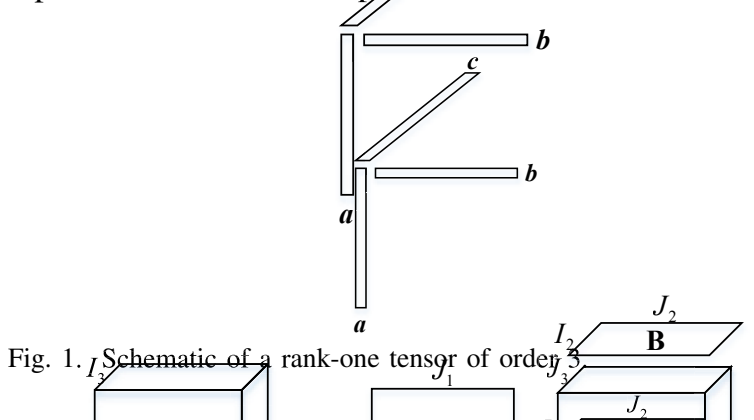

Definition 7. The $=$ rank of atens $\phi r y$ 2the

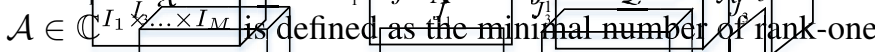

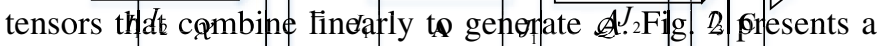
3-way tensor of rank three. $I_{2}$

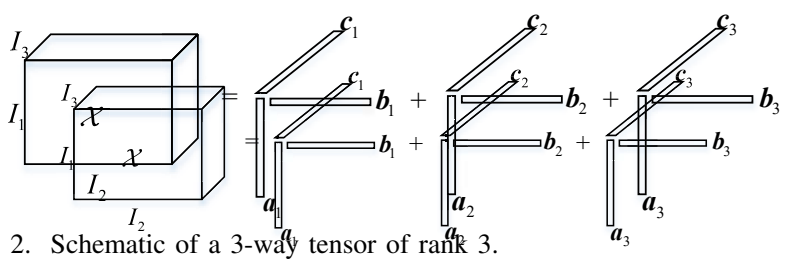

Fig. 2. Schematic of a 3-way tensor of rank 3 .

Definition 8. Karank: The rank $r a$ of $\mathbf{A} \in \mathbb{C}_{\mathscr{C}^{R}}^{I^{\times} \times J}$ is equal to $r$ if A contains at least 2 st $t_{b}$ of 1 nearly. indendent columns but no set of $x^{2}+1$ line th independent columns. The Kruskal-

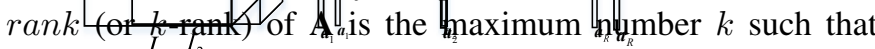
every set of $k^{2}$ columns of $\mathbf{A}$ is linearly independent. Note that the $k$-rank is always less than or equal to $r_{\mathbf{A}}$. That is,

$$
k_{\mathbf{A}} \leq r_{\mathbf{A}} \leq \min (I, J) \text {. }
$$

\section{B. Tensor Decompositions}

1) Tucker decomposition: The Tucker decomposition decomposes a tensor into a core tensor of the same order and some factor matrices. For an $M$ th order tensor $\mathcal{A} \in$ $\mathbb{C}^{I_{1} \times \ldots \times I_{M}}$, the Tucker decomposition is defined as [10]

$$
\mathcal{A}=\mathcal{Q} \times{ }_{1} \mathbf{X}^{(1)} \times{ }_{2} \mathbf{X}^{(2)} \cdots \times_{M} \mathbf{X}^{(M)},
$$

where $\mathcal{Q} \in \mathbb{C}^{J_{1} \times \ldots \times J_{M}}$ is the core tensor and $\mathbf{X}^{(m)} \in$ $\mathbb{C}^{I_{m} \times J_{m}}$, with $m=1, \cdots, M$, are the factor matrices. The elements of $\mathcal{A}$ can be represented as

$$
a_{i_{1}, \ldots, i_{M}}=\sum_{j_{1}=1}^{J_{1}} \ldots \sum_{J_{M}=1}^{J_{M}} q_{j_{1}, \ldots, j_{M}}^{c} \prod_{m=1}^{M} x_{i_{m}, j_{m}}^{(m)} .
$$

For illustration, we show in Fig. 3 the Tucker decomposition of a third-order tensor $\mathcal{X} \in \mathbb{C}^{I_{1} \times I_{2} \times I_{3}}$ as $\mathcal{X}=\mathcal{Q} \times{ }_{1} \mathbf{A} \times{ }_{2}$ $\mathbf{B} \times{ }_{3} \mathbf{C}$, where $\mathcal{Q} \in \mathbb{C}^{J_{1} \times J_{2} \times J_{3}}$ is the core tensor and the factor matrices are dembted by $\mathbf{A} \in \mathbb{C}^{I_{1} \times J_{1}}, \mathbf{B} \in \mathbb{C}^{I_{2} \times J_{2}}$ and $\mathbf{C} \in \mathbb{C}^{I_{3} \times J_{3}}$

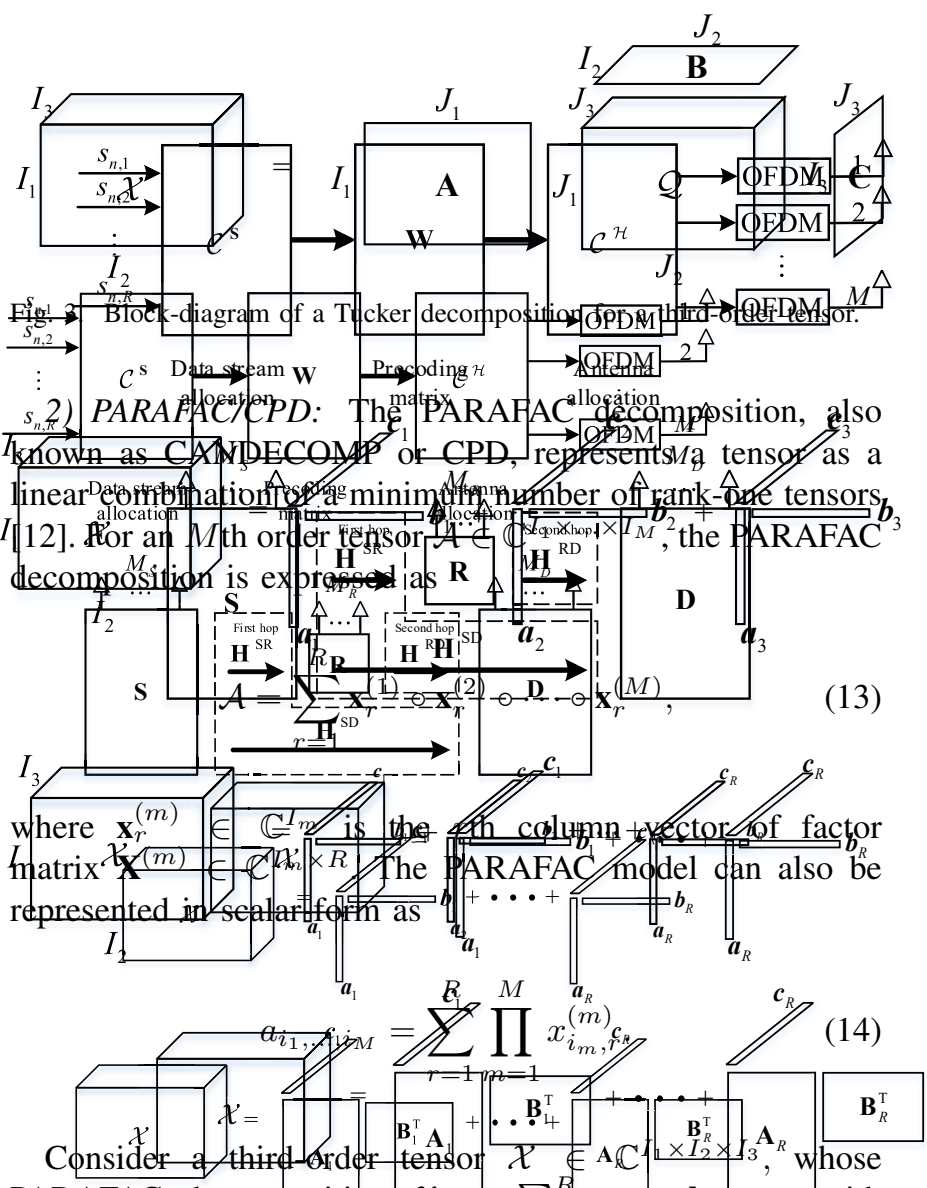

PARAFAC decomposition $\mathcal{X} \equiv \sum_{r}^{R} \mathbf{a}_{1} \mathbf{a}_{r} \circ \mathbf{b}_{r} \circ \mathbf{c}_{r}$ with $\mathbf{A} \in \mathbb{C}^{I_{1} \times R}, \mathbf{B} \in \mathbb{C}^{I_{2} \times R}$ and $\mathbf{C} \in \mathbb{C}^{I_{3} \times R}$. We can obtain $\mathbf{X}_{:, i_{2},:}=\sum_{r=1}^{R} b_{i_{2}, r} \mathbf{c}_{r} \mathbf{a}_{r}^{T}=\mathbf{C} D_{i_{2}}(\mathbf{B}) \mathbf{A}^{T} \in \mathbb{C}^{I_{3} \times I_{1}}$
$\mathbf{X}_{:,:, i_{3}}=\sum_{r=1}^{R} b_{i_{3}, r} \mathbf{a}_{r} \mathbf{b}_{r}^{T}=\mathbf{A} D_{i_{3}}(\mathbf{C}) \mathbf{B}^{T} \in \mathbb{C}^{I_{1} \times I_{2}}$ for $i_{1}=1, \cdots, I_{1}, i_{2}=1, \cdots, I_{2}$ and $i_{3}=1, \cdots, I_{3}$. The notation : is derived from the Matlab notations. Stacking these 

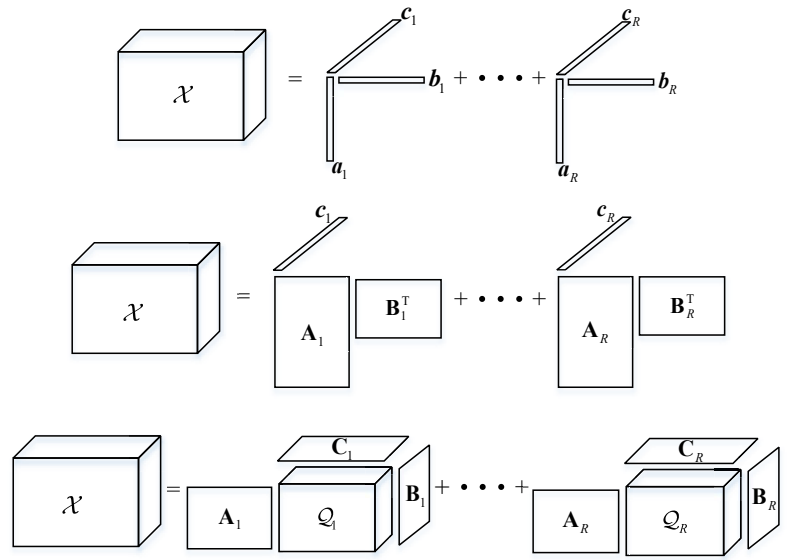

Fig. 4. Illustration of BTD models. Top: rank- $(1,1,1)$ BTD or CPD; Middle: rank- $(L, L, 1)$ BTD. Bottom: rank- $(L, M, N)$ BTD.

slices columnwise, we obtain three possible unfoldings of the tensor $\mathcal{X}$ as

$$
\begin{aligned}
& \mathbf{X}_{I_{1} I_{2} \times I_{3}}= {\left[\begin{array}{c}
\mathbf{B} D_{1}(\mathbf{A}) \\
\vdots \\
\mathbf{B} D_{I_{2}}(\mathbf{A})
\end{array}\right] \mathbf{C}^{T}=(\mathbf{A} \diamond \mathbf{B}) \mathbf{C}^{T}, } \\
& \mathbf{X}_{I_{2} I_{3} \times I_{1}}= {\left[\begin{array}{c}
\mathbf{C} D_{1}(\mathbf{B}) \\
\vdots \\
\mathbf{C} D_{I_{3}}(\mathbf{B})
\end{array}\right] \mathbf{A}^{T}=(\mathbf{B} \diamond \mathbf{C}) \mathbf{A}^{T}, } \\
& \mathbf{X}_{I_{3} I_{1} \times I_{2}}=\left[\begin{array}{c}
\mathbf{A} D_{1}(\mathbf{C}) \\
\vdots \\
\mathbf{A} D_{I_{1}}(\mathbf{C})
\end{array}\right] \mathbf{B}^{T}=(\mathbf{C} \diamond \mathbf{A}) \mathbf{B}^{T} .
\end{aligned}
$$

3) Block term decomposition: Block term decomposition (BTD) aims to find components with specific multilinear rank terms. The BTD framework was first introduced in [65], where the CPD and Tucker decomposition can be considered as special cases in this framework. For simplicity, we focus on third-order tensors in this subsection. The rank-1 term in CPD can be viewed as the multilinear rank- $(1,1,1) \mathrm{BTD}$, while the Tucker decomposition can be regarded as the multilinear rank$(L, M, N)$ BTD with only one term; see Fig. 4 for different types of BTD. For a detailed description of additional BTD variants, the reader is referred to [66], [67].

The decomposition of 3-way tensors in rank- $(L, L, 1)$ terms finds many applications in psychometrics, chemometrics, neuroscience, and signal processing, similar to its CPD counterpart. Rank- $(L, L, 1)$ BTD is essentially unique under some mild conditions and its factors have explicit physical interpretations, which has been proven useful for blind source separation [68], [69] in array signal processing [73], spectrum cartography [74], and hyperspectral super-resolution (HSR) [75]. Formally, the rank- $(L, L, 1)$ BTD of a tensor $\mathcal{X} \in$ $\mathbb{R}^{I \times J \times K}$ is a decomposition of the form

$$
\mathcal{X}=\sum_{r=1}^{R} \mathbf{E}_{r} \circ \mathbf{c}_{r},
$$

where $\mathbf{E}_{r}=\mathbf{A}_{r} \mathbf{B}_{r}^{T} \in \mathbb{R}^{I \times J}$ is a rank- $L$ matrix, $\mathbf{A}_{r} \in \mathbb{R}^{I \times L}$, $\mathbf{B}_{r} \in \mathbb{R}^{J \times L}$, and $\mathbf{c}_{r} \in \mathbb{R}^{K}$. The factors $\left\{\mathbf{A}_{r}, \mathbf{B}_{r}, \mathbf{c}_{r}\right\}$ can be determined using alternating least squares (ALS), gradientbased methods, and nonlinear least squares (NLS) [66], [67].

4) Uniqueness: The Tucker model is not essentially unique [76]. This is because the factor matrices $\mathbf{X}^{(m)}$ and the core tensor $\mathcal{Q}$ are not uniquely identifiable. More specifically, $\mathbf{X}^{(m)}$ and $\mathcal{Q}$ can be replaced by $\hat{\mathbf{X}}^{(m)}=\mathbf{X}^{(m)} \Delta_{m}$ and $\hat{\mathcal{Q}}=\mathcal{Q} \times{ }_{m=1}^{M}$ $\left(\Delta_{m}\right)^{-1}$, respectively, with $\Delta_{m} \in \mathbb{C}^{J_{m} \times J_{m}}$ being nonsingular, without changing the tensor $\mathcal{A}$. That is,

$$
\begin{aligned}
\mathcal{A} & =\hat{\mathcal{Q}} \times{ }_{m=1}^{M} \hat{\mathbf{X}}^{(m)} \\
& =\mathcal{Q} \times{ }_{m=1}^{M}\left(\Delta_{m}\right)^{-1} \times_{m=1}^{M} \mathbf{X}^{(m)} \Delta_{m} \\
& =\mathcal{Q} \times{ }_{m=1}^{M} \mathbf{X}^{(m)} \Delta_{m}\left(\Delta_{m}\right)^{-1} \\
& =\mathcal{Q} \times{ }_{m=1}^{M} \mathbf{X}^{(m)}
\end{aligned}
$$

This implies that the core tensor and the factor matrices have alternatives which satisfy the decomposition model.

As opposed to the Tucker model, the PARAFAC/CPD is essentially unique, i.e., the factor matrices $\mathbf{X}^{(m)}$ in (13) are unique provided the following sufficient condition is satisfied [77]

$$
\sum_{m=1}^{M} k_{\mathbf{X}^{(m)}} \geq 2 R+(M-1)
$$

where $k_{\mathbf{X}^{(m)}}$ is the $k$-rank of the factor matrices $\mathbf{X}^{(m)} \in$ $\mathbb{C}^{I_{m} \times R}$. The matrices $\mathbf{X}^{(m)}, m=1, \ldots, M$, are unique up to permutation and (complex) scaling of its columns [23], [81]. This means that every set of matrices $\tilde{\mathbf{X}}^{(m)}$ satisfying (15)-(17) is linked to $\mathbf{X}^{(m)}$ by

$$
\tilde{\mathbf{X}}^{(m)}=\mathbf{X}^{(m)} \Pi \Delta_{m}, m=1, \ldots, M,
$$

where $\Pi$ is a permutation matrix and $\left\{\Delta_{m}\right\}_{m=1}^{M}$ are diagonal matrices satisfying the condition

$$
\prod_{m=1}^{M} \Delta_{m}=\mathbf{I}_{R}
$$

with $\mathbf{I}_{R} \in \mathbb{R}^{R \times R}$ being an identity matrix.

The PARATUCK decomposition is a very powerful decmposition and has found inumerous applications. It combines the properties of the PARAFAC and Tucker decompositions, which makes it more flexible to model different communication models that are not captured by PARAFAC models, while affording uniqueness under mild conditions [79], [80]. Take PARATUCK2 model as an example, the element for a third-order tensor $\mathcal{X} \in \mathbb{R}^{I_{1} \times I_{2} \times I_{3}}$ is defined as

$$
x_{i_{1}, i_{2}, i_{3}}=\sum_{m=1}^{M} \sum_{r=1}^{R} a_{i_{1}, m} b_{i_{2}, r} g_{m, r} c_{i_{3}, m}^{(\mathbf{A})} c_{i_{3}, r}^{(\mathbf{B})},
$$

where $x_{i_{1}, i_{2}, i_{3}}$ is the $\left(i_{1}, i_{2}, i_{3}\right)$-th entry of $\mathcal{X}, \mathbf{A} \in \mathbb{C}^{I_{1} \times M}$, $\mathbf{B} \in \mathbb{C}^{I_{2} \times R}, \mathbf{C}^{(\mathbf{A})} \in \mathbb{C}^{I_{3} \times M}, \mathbf{C}^{(\mathbf{B})} \in \mathbb{C}^{I_{3} \times R}$. The matrices $\mathbf{A}$ and $\mathbf{B}$ are associated with the first and second dimensions of $\mathcal{X} . \mathbf{C}^{(\mathbf{A})}$ and $\mathbf{C}^{(\mathbf{B})}$ are interaction matrices defining the linear combination profile between the $M$ columns of $\mathbf{A}$ and the $R$ columns of $\mathbf{B}$ along the third dimension of $\mathcal{X}$. $\mathbf{G}$ is the core matrix of the PARATUCK2 model. The element $g_{m, r}$ of $\mathbf{G}$ defines the magnitude of the interaction between the $m$-th column of $\mathbf{A}$ and the $r$-th column of $\mathbf{B}$. 
Similar to CPD, rank- $(L, L, 1)$ BTD also has essential uniqueness. One can arbitrarily permute the different rank$(L, L, 1)$ terms. Within a rank- $(L, L, 1)$ term, the factors $\mathbf{E}_{r}$ and $\mathbf{c}_{r}$ can be arbitrarily scaled, as long as their product remains the same. The factors $\mathbf{A}_{r}, \mathbf{B}_{r}$ can be multiplied by any nonsingular matrix $\mathbf{F}_{r} \in \mathbb{R}^{L \times L}$ provided that $\mathbf{A}_{r} \mathbf{F}_{r}\left(\mathbf{B}_{r} \mathbf{F}_{r}^{-T}\right)^{T}=\mathbf{A}_{r} \mathbf{B}_{r}^{T}$. A rank- $(L, L, 1)$ BTD is said to be essentially unique if it is subject to these trivial ambiguities. Necessary and sufficient conditions of essential uniqueness can be found in [66], [68].

Finally, it is worth mentioning that the tensor decomposition techniques based on the data fitting principle have been extended/generalized in several ways. One important extension is to robust PARAFAC, where a trilinear alternating least absolute error minimization substitutes trilinear alternating least squares minimization [70]. It provides the robustness to outliers (non-Gaussian error), which is a common problem in, for example, multi-user communication systems with multiple interferes as well as jammer suppression and clutter mitigation in radar. Another recent extension is sparse tensor decomposition [71], [72] that ensures the sparsity of the decomposition and has potential applications in, for example, millimeter wave channel estimation, which will be reviewed latter.

\section{Applications in Wireless Communications}

In this section, we provide an overview of tensor analysis in wireless communication. We group tensor-based methods on the nature of the signal processing tasks undertaken and system types.

\section{A. Blind Multiuser CDMA}

We consider a typical uplink direct-sequence CDMA (DSCDMA) communication system having one base station (BS) and $M$ users. Let $K$ antennas be mounted on the BS. The spreading code of user $m$ is denoted by $\mathbf{c}_{m}=$ $\left[c_{m}(1), c_{m}(2), \cdots, c_{m}(L)\right]^{T} \in \mathbb{C}^{L \times 1}$, with $L$ being the spreading gain and $c_{m}(l)$ representing its $l$ th chip. The $n$th transmitted symbol from user $m$ is $s_{m}(n)$. The fading/path loss between the BS and the user $m$ is denoted as $\mathbf{h}_{m}=$ $\left[h_{m}(1), h_{m}(2), \cdots, h_{m}(K)\right]^{T} \in \mathbb{C}^{K \times 1}$. Then, the baseband output for symbol $n$ and chip $l$ from the $k$ th antenna can be expressed as

$$
x_{k, n, l}=\sum_{m=1}^{M} h_{m}(k) c_{m}(l) s_{m}(n) .
$$

We define $K \times N$ data matrices as

$$
\mathbf{X}_{l} \triangleq\left[\begin{array}{cccc}
x_{1,1, l} & x_{1,2, l} & \cdots & x_{1, N, l} \\
x_{2,1, l} & x_{2,2, l} & \cdots & x_{2, N, l} \\
\vdots & \vdots & \vdots & \vdots \\
x_{K, 1, l} & x_{K, 2, l} & \cdots & x_{K, N, l}
\end{array}\right]
$$

for $l=1, \cdots, L$. With some mathematical manipulation, we can show that $\mathbf{X}_{l}$ satisfies the factorization

$$
\mathbf{X}_{l}=\mathbf{H} D_{l}(\mathbf{C}) \mathbf{S}^{T}
$$

where

$$
\begin{gathered}
\mathbf{H} \triangleq\left[\begin{array}{cccc}
h_{1}(1) & h_{2}(1) & \cdots & h_{M}(1) \\
h_{1}(2) & h_{2}(2) & \cdots & h_{M}(2) \\
\vdots & \vdots & \vdots & \vdots \\
h_{1}(K) & h_{2}(K) & \cdots & h_{M}(K)
\end{array}\right] \\
\mathbf{C} \triangleq\left[\begin{array}{cccc}
c_{1}(1) & c_{2}(1) & \cdots & c_{M}(1) \\
c_{1}(2) & c_{2}(2) & \cdots & c_{M}(2) \\
\vdots & \vdots & \vdots & \vdots \\
c_{1}(L) & c_{2}(L) & \cdots & c_{M}(L)
\end{array}\right] \\
\mathbf{S} \triangleq\left[\begin{array}{cccc}
s_{1}(1) & s_{2}(1) & \cdots & s_{M}(1) \\
s_{1}(2) & s_{2}(2) & \cdots & s_{M}(2) \\
\vdots & \vdots & \vdots & \vdots \\
s_{1}(N) & s_{2}(N) & \cdots & s_{M}(N)
\end{array}\right] .
\end{gathered}
$$

With the factorization in PARAFAC model, the spreading codes $\mathbf{C} \in \mathbb{C}^{L \times M}$, information symbols $\mathbf{S} \in \mathbb{C}^{M \times N}$, and the path fading loss $\mathbf{H} \in \mathbb{C}^{K \times M}$ can be recovered as long as the decomposition uniqueness condition is satisfied. We note that the model in (29) does not consider practical constraints, such as frequency-selective channel, time synchronization issues, MIMO case, etc.

The work [23] was the first to introduce tensor model for signal processing in wireless communication systems. The authors proposed a blind PARAFAC model-based separationequalization-detection receiver for DS-CDMA multiuser systems. The blind receiver was shown to achieve the same performance as that of the non-blind minimum mean-squared error (MMSE) receiver. Owing to the fact that it did not require statistical independence or knowledge of the codes, the receiver offered a higher flexibility for incorporation into different types of systems compared to earlier approaches.

The authors in [25] unified the received signal model of three multiuser systems, namely, a temporally oversampled system, a DS-CDMA system, and an OFDM system, into a tensor (3-D) PARAFAC model. Each considered multiuser system employed multiple antennas at the receiver and was assumed to be subject to frequency-selective multipath fading. A new tensor-based receiver was designed that performed, in an iterative fashion, multiuser signal separation by determining the PARAFAC model parameters and signal equalization via subspace methods. Simulation results showed that the tensorbased receiver provided performance close to the MMSE solution with a perfect knowledge of the propagation parameters.

The work [26] proposed a two-stage blind tensor based detector for the uplink communication of a wideband-CDMA (W-CDMA) system subject to large delay spread. The lowrank decomposition of the 3-D received data enabled an Finite Impulse Response-MIMO (FIR-MIMO) CDMA problem to be converted into multiple standard independent FIR single-input multiple-output problems; the latter were solved using known techniques, such as the Hankel kernel approach. The paper [28], on the other hand, devised a constrained tensor modeling approach for an uplink CDMA communication system where the BS and the users employed multiple antennas. Two constraint matrices were considered to respectively control the spatial spreading of the data streams and the spatial reuse of 
the spreading codes. A systematic design procedure for the canonical allocation matrices was developed which derived a finite set of multiple-antenna schemes for a fixed number of transmit antennas. Identifiability of the proposed tensor model was also determined to guarantee blind symbol recovery.

\section{B. Space-Time Frequency (STF) MIMO Systems}

In wireless communication systems, incorporation of oversampling, spreading, multiplexing, diversity, and other operations yields multi-dimensional received signals, for which the tensor models are a natural fit. We consider a typical multi-carrier MIMO wireless communication system, where the BS uses $M$ transmit antennas, $R$ data streams, and $F$ subcarriers. A total of $K$ receive antennas are employed. We assume the transmission to be decomposed into $P$ data blocks, with each block consisting of $N$ symbol periods. The transmitted symbols are spread and multiplexed in space (multiple antennas), time (time blocks and time spreading), and frequency (multi-carriers) domains. We denote by $s_{n, r}$ the $n$th symbol of the $r$ th data stream, and $\mathbf{W} \in \mathbb{C}^{M \times R}$ is the coding matrix that maps the signals from the data streams to the antennas.

For a fixed symbol period $p$ and subcarrier $f$, the $(m, p, f)$ th STF coded signal associated with the $m$ th transmit antenna, $p$ th block, and $f$ th subcarrier is generated by two allocation tensors, namely, the stream allocation tensor $\mathcal{C}^{(\mathbf{S})} \in \mathbb{R}^{F \times P \times R}$ and the antenna allocation tensor $\mathcal{C}^{(\mathcal{H})} \in \mathbb{R}^{F \times P \times M}$. The former determines the time-frequency mapping of the $R$ data streams across $P$ blocks and $F$ subcarriers, while the latter disctates the time-frequency mapping of the $M$ transmit antennas. The elements of both tensors assume a value equal to zero or unity.

The coded signal can be formed into a fourth-order tensor $\mathcal{U} \in \mathbb{C}^{F \times M \times N \times P}$, where the $(f, m, n, p)$ th element corresponds to the $f$ th subcarrier, $m$ th transmit antenna, $n$th symbol period, and $p$ th data block, and is given by

$$
\begin{aligned}
u_{f, m, n, p} & =\sum_{r=1}^{R} w_{m, r} s_{n, r} c_{f, p, m}^{(\mathcal{H})} c_{f, p, r}^{(\mathbf{S})} \\
& =\sum_{r=1}^{R} t_{f, m, r, p} s_{n, r},
\end{aligned}
$$

with

$$
t_{f, m, r, p} \triangleq w_{m, r} c_{f, p, m}^{(\mathcal{H})} c_{f, p, r}^{(\mathbf{S})} .
$$

We define $\mathcal{H} \in \mathbb{C}^{F \times K \times M}$ as the channel tensor for the MIMO-OFDM communication system. The fading coefficients are assumed to be constant during $P$ blocks. For the noiseless case, the received signal tensor $\mathcal{X} \in \mathbb{C}^{F \times K \times N \times P}$, corresponding to the $f$ th subcarrier and received at the $k$ th antenna during the $n$th symbol period of the $p$ th data block, is given by

$$
\begin{aligned}
x_{f, k, n, p} & =\sum_{m=1}^{M} h_{f, k, m} u_{f, m, n, p} \\
& =\sum_{m=1}^{M} \sum_{r=1}^{R} t_{f, m, r, p} h_{f, k, m} s_{n, r} .
\end{aligned}
$$

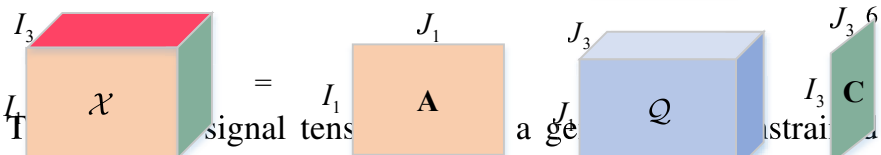

PARATUCK-2 model. Using the mode- $\iota$ pryuun definition from (8), we can write the $f$ th tensorial slice of $\mathcal{X} \in$ $\mathbb{C}^{F \times K \times N \times P}$, containing all received signals associated with the subcarrier $f$, as
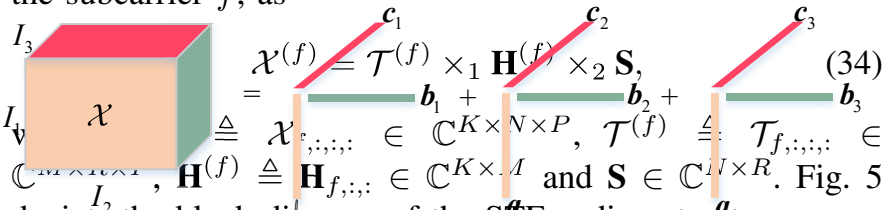

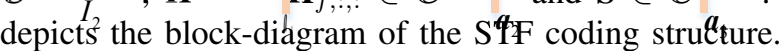

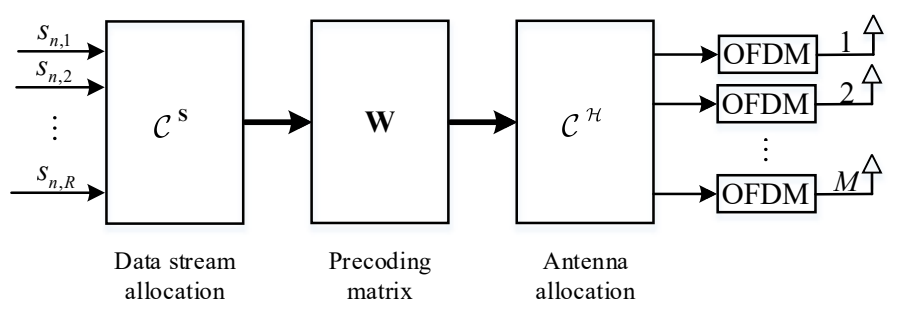

Fig. 5. SFT coding structure.

By introducing extra diversity into the transmitting signal, e.g., space, time, frequency and code, the tensor modeling is able to encode the signal and exploit the coding features. Following [23], the flexible class of Khatri-Rao Space-Time (KRST) codes based on the PARAFAC tensor model was also developed in [34]. The work [35] combines space-frequency spreding and multiplexing functionalities for MIMO multistream multi-carrier systems, while allowing a semi-blind joint channel estimation and detection using the PARAFAC model.

A new tensorial approach based on a tensor space-time (TST) coding was proposed in [36] for MIMO wireless communication systems. The received signals assumed the form of a fourth-order tensor which was shown to satisfy a PARATUCK- $\left(N_{1}, N\right)$ model with $N_{1}=2$ and $N=4$. The uniqueness conditions for the PARATUCK- $\left(N_{1}, N\right)$ model were also established therein. Semi-blind receivers based on ALS, Levenberg-Marquardt (LM) and the Kronecker Least Squares (KLS) methods were designed for both the TST and STF systems.

In [37], a transmission scheme based on two allocation matrices for selection of antennas and data streams was modeled utilizing only the space and time domains. A generalized fourth-order PARATUCK2 tensor model for MIMO communications with STF spreading-multiplexing was proposed in [38]. The core of this fourth-order tensor was essentially composed of two third-order interaction tensors. Data streams (multiplexing degree) and transmit antennas (space) were allocated to time blocks (time) and subcarriers (frequency). A blind receiver based on LM algorithm was proposed for the generalized fourth-order PARATUCK-2 model.

The authors in [39] proposed two new classes of constrained tensor models, called the generalized PARATUCK- $\left(N_{1}, N\right)$ model and the generalized Tucker- $\left(N_{1}, N\right)$ model, with highorder tensors being the factors of the decompositions. A new tensor STF coding which led to a generalized PARATUCK$(2,5)$ model was proposed for MIMO OFDM-CDMA systems. Two semi-blind receivers, one iterative and the other in closed- 
form, were proposed for a joint channel and symbol estimation. The former was based on a two-step ALS algorithm, while the latter comprised a low-complexity solution based on Kronecker product least squares estimation.

In [40], a two-step tensor-based receiver based on the fourthorder PARATUCK2 model was proposed for a modified spacetime coding scheme which incorporated a formatting filter. In the first step, closed-form channel estimation was performed by means of Kronecke $\boldsymbol{e}_{2}$ and Khatri-Rao factorizations. In the second step, the trans ${ }^{2}$ tted symbols we linearly decoded by exploiting the est mated channel. Simulation results demonstrated $\mid$ the effectivanos of $b_{2}$ he tencor_hased receiver in terms of normalized nean squared errc $r$ and bit errơ rate (BER).

\section{Cooperatila 2 Relay Systems $\boldsymbol{a}_{3}$}

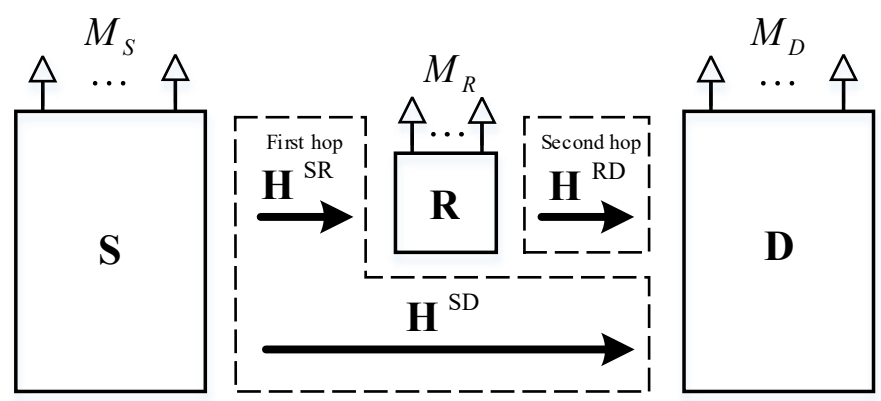

Fig. 6. Two-hop relay communication system.

We consider a typical amplify-and-forward (AF) two-hop cooperative system with a source node $(\mathrm{S})$, a destination node (D), and a relay (R), as shown in Fig. 6. The respective numbers of antennas at the source, destination, and the relay are assumed to be $M_{S}, M_{D}$ and $M_{R}$. Matifices $\mathbf{H}^{S D} \in \mathbb{C}^{M_{D} \chi_{R} M_{S}}$, $\mathbf{H}^{S R} \in \mathbb{C}^{M_{R} \times M_{S}}$ and $\mathbf{H}^{R D} \in \mathbb{C}^{M b \times M_{R}}$ denote the channel of the direct link $\overline{\overline{\text { to }}}$ destination ${ }^{+}$(SD link), the channel between the source and the relay (SR link), and the channel between the relay and the destination ( $R D$ link), respectively. Let $\mathbf{S} \notin_{2} \mathbb{C}^{N \times M_{S}}$ be the information symbol matrix during $N$ timeblocks. A code matrix $\mathbf{C} \in \mathbb{C}^{P \times M_{S}}$ is used, where $P$ is the number of symbol periods in a time block. The transmitted signal at the $n$th time block is given by

$$
\mathbf{X}_{n}=D_{n}(\mathbf{S}) \mathbf{C}^{T} \in \mathbb{C}^{M_{S} \times P}
$$

1) Model of the SD Link: The $n$th received signal is given by

$$
\mathbf{Y}_{:,:, n}^{(S D)}=\mathbf{H}^{(S D)} \mathbf{X}_{n}=\mathbf{H}^{(S D)} D_{n}(\mathbf{S}) \mathbf{C}^{T} \in \mathbb{C}^{M_{D} \times P} .
$$

where $\mathbf{Y}_{:,, n}$ is the $n$th slice of the third-order received signal tensor $\mathcal{Y}^{(S D)} \in \mathbb{C}^{M_{D} \times P \times N}$. Eq. (36) represents a PARAFAC decomposition of $\mathcal{Y}^{(S D)}$. The elements of $\mathcal{Y}^{(S D)}$ can be expressed as

$$
y_{m_{D}, p, n}^{(S D)}=\sum_{m_{S}=1}^{M_{S}} h_{m_{D}, m_{S}}^{S D} c_{p, m_{S}} s_{n, m_{S}} .
$$

Three unfolded forms of this PARAFAC model are given by

$$
\begin{aligned}
\mathbf{Y}_{P N \times M_{D}}^{(S D)} & =(\mathbf{S} \diamond \mathbf{C})\left(\mathbf{H}^{(S D)}\right)^{T}, \\
\mathbf{Y}_{N M_{D} \times P}^{(S D)} & =\left(\mathbf{H}^{(S D)} \diamond \mathbf{S}\right)(\mathbf{C})^{T}, \\
\mathbf{Y}_{M_{D} P \times N}^{(S D)} & =\left(\mathbf{C} \diamond \mathbf{H}^{(S D)}\right)(\mathbf{S})^{T} .
\end{aligned}
$$

2) Model of the Source-Relay-Destination (SRD) Link: The signals received at the relay are first stored and then an $\mathrm{AF}$ matrix $\mathbf{G} \in \mathbb{C}^{N \times M_{R}}$ is used to code the stored signals, which are transmitted through the channel $\mathbf{H}^{(R D)}$. The received signals at the destination via the relay form a third-order tensor $\mathcal{Y}^{(S R D)} \in \mathbb{C}^{M_{D} \times P \times N}$ whose $n$th slice is given by

$$
\mathbf{Y}_{:,:, n}^{(S R D)}=\mathbf{H}^{(R D)} D_{n}(\mathbf{G}) \mathbf{H}^{(S R)} D_{n}(\mathbf{S}) \mathbf{C}^{T} \in \mathbb{C}^{M_{D} \times P} .
$$

Eq. (41) represents a PARATUCK2 decomposition of the tensor $\mathcal{Y}^{(S R D)}$, whose elements can be written as

$$
y_{m_{D}, p, n}^{(S R D)}=\sum_{m_{R}=1 m_{S}=1}^{M_{R}} \sum_{m_{S}, m_{R}}^{M_{S}} h_{n, m_{R}}^{(R D)} h_{m_{R}, m_{S}}^{(S R)} c_{p, m_{S}} s_{n, m_{S}} .
$$

In the PARAFAC and PARATUCK2 decompositions, the uniqueness can be established under specific conditions. Both semi-blind and blind receivers have been investigated to estimate the channels, $\mathbf{H}^{(S R)}$ and $\mathbf{H}^{(D R)}$, and the symbol $\mathbf{S}$ by means of algorithms, such as the ALS. In cooperative systems, the system reliability strongly depends on the accuracy of channel state information (CSI) of all links between the source, relays and destination. Under the assumption that CSI is not available, the authors in [82] proposed a blind receiver model for uplink multiuser cooperative diversity systems by exploiting a unified formulation of the received signals as a PARAFAC model. Three relay protocols were considered, namely, AF, fixed decode-and-forward, and selective decodeand-forward. In [83], a simplified KRST coding was adopted at the transmission source in a two-hop AF cooperative scheme. Three different receivers using the PARAFAC and PARATUCK2 models for the SD and SRD links were designed. Simulation results demonstrated the superior performance of these receivers over supervised approaches in terms of the BER.

In [84], the author proposed the nested Tucker decomposition (NTD) model. Then, by exploiting a tensor space-time coding (TSTC) structure at both the source and the relay nodes in a one-way two-hop MIMO relay communication system, an NTD of the fourth-order tensor was formed at the destination. Two semi-blind receivers and two supervised receivers were derived to jointly estimate the transmitted information symbols and the two individual relay channels. The authors in [85] performed joint channel estimation for a three-hop MIMO system with an AF relaying protocol. ALS algorithm was used by coupling PARAFAC and Tucker3 tensor models for the received signals to iteratively estimate the channel matrices. Simulation results corroborated the effectiveness of the joint channel estimator when compared to two sequential estimators. In [86], a one-way multi-hop AF relaying system was modeled based on a generalized nested PARAFAC decomposition. Under the assumption of 
KRST coding implemented at each relay, a sequential closedform semi-blind receiver was designed, wherein the information symbols and the individual channels were jointly estimated. For OFDM-based cooperative communication systems, a tensor-based blind signal recovery scheme was devised in [87]. The received multi-carrier signals were modeled as a 3$\mathrm{D}$ tensor and a PARAFAC decomposition-based blind receiver algorithm was employed for data detection. The work in [88] proposed two ST coding schemes, MKRST and MKronST, for multiple-antenna one-way two-hop MIMO relay system. These coding schemes generalize the standard Khatri-Rao coding by introducing extra space/time diversities. Parallel non-iterative decoding methods are proposed for estimating the symbol matrix. In [89], the authors considered the multirelaying systems, two tensor-based receivers were proposed to jointly estimate the channels and symbols in a semi-blind fashion. By exploiting the structure of the received signals, the authors shown that the data for the relay-assisted link after ST decoding has a Kronecker structure, which can be recast as a rank-one tensor based on PARAFAC analysis. The simulation results also shown that the proposed receiver design has good performance-complexity trade-off.

\section{Time-varying Channel Modeling}

Tensor decompositions were considered for on-line applications in [90], where the data were assumed to be serially acquired and/or the underlying model was considered to change frequently, resulting in a time-varying wireless communication system. Given the PARAFAC decomposition of a tensor at instant $t$, two adaptive low-complexity algorithms were provided to obtain the decomposition at instant $t+1$ by appending a new slice in the time dimension, and their excellent tracking capability was validated through simulation.

Doppler shifts in a time-varying mmWave scenario were considered in [91]. The channel was assumed to have blocksparse and low-rank characteristics, since the change in angle was much slower than that in path gain. By exploiting these characteristics, a two-stage method was proposed. In the first stage, Block Orthogonal matching pursuit (BOMP) algorithm was used to estimate the angles-of-arrival (AoAs)/angles-ofdeparture (AoDs). Based on the angle estimates, PARAFAC decomposition was used to estimate the Doppler shifts and path gains in the second stage. Furthermore, the proposed algorithm was shown to be close to the Cramer-Rao Lower Bound (CRLB). For downlink multiuser-MIMO (MU-MIMO) communications over a time-varying channel, a transmission frame structure was proposed in [92], wherein the angle and the channel gain were to be estimated. By leveraging the sparse nature of mmWave channels, an adaptive angle estimation algorithm was devised. The angle estimates were used to design pilot beamforming for estimating the path gains. For the same assumption of slower variations in angle than path gains, the authors in [93] proposed a two-stage tensor decomposition based method for a single receiver. Doppler shift estimation was achieved based on the estimated angles.

\section{E. mmWave Communication System}

Millimeter wave channels have a sparse scattering nature, leading to their low-rank structures and spread in the form of clusters of paths over the angular domains, including the AoA, AoD, and elevation. This joint sparse and low-rank structure renders the application of tensor models suitable in mmWave communication systems.

Consider a point-to-point uplink mmWave MIMO system, which comprises $N$ antennas at the BS and $M$ antennas at the MS. Beamforming techniques are implemented due to short wavelength and severe path loss in mmWave communication system. Analog beamforming is used in both BS and MS with one radio-frequency chain. At time instant $t$, the symbol $s(t)$ is transmitted through an analog beamforming vector $\mathbf{f}(t) \in \mathbb{C}^{N}$. The receiver combines the signals with receive beamforming vector $\mathbf{w}(t) \in \mathbb{C}^{M}$. The combined signal at the receiver can be expressed as

$$
y(t)=\mathbf{w}^{H}(t) \mathbf{H f}(t) s(t)+\mathbf{w}^{H}(t) n(t) \quad \forall t=1, \ldots, T,
$$

where $\mathbf{H} \in \mathbb{C}^{M \times N}$ is the channel matrix and $n(t)$ denotes additive Gaussian noise. For mmWave operation, the channel is usually characterized by a geometric model [91]

$$
\mathbf{H}=\sum_{l=1}^{L} \alpha_{l} \mathbf{a}_{B S}\left(\theta_{l}\right) \mathbf{a}_{M S}^{H}\left(\psi_{l}\right),
$$

where $L$ is the number of paths, $\alpha_{l}$ is the complex gain associated with the $l$ th path, and $\theta_{l}$ and $\psi_{l}$ are the AoA and AoD, respectively. The vectors $\mathbf{a}_{B S}$ and $\mathbf{a}_{M S}$ represent the array response vectors and are given by

$$
\begin{aligned}
\mathbf{a}_{B S}\left(\theta_{l}\right) & =\frac{1}{\sqrt{N}}\left[1, e^{j \frac{2 \pi}{\lambda} d \sin \left(\theta_{l}\right)}, \ldots, e^{j(N-1) \frac{2 \pi}{\lambda} d \sin \left(\theta_{l}\right)}\right]^{T} \\
\mathbf{a}_{M S}\left(\psi_{l}\right) & =\frac{1}{\sqrt{M}}\left[1, e^{j \frac{2 \pi}{\lambda} d \sin \left(\psi_{l}\right)}, \ldots, e^{j(N-1) \frac{2 \pi}{\lambda} d \sin \left(\psi_{l}\right)}\right]^{T} .
\end{aligned}
$$

The channel matrix can be further formulated as

$$
\mathbf{H}=\mathbf{A}_{B S} \mathbf{H}_{v} \mathbf{A}_{M S}^{H}
$$

where $\mathbf{A}_{B S} \triangleq\left[\mathbf{a}_{B S}\left(\phi_{1}\right), \ldots, \mathbf{a}_{B S}\left(\phi_{N_{1}}\right)\right] \in \mathbb{C}^{N \times N_{1}}$ is the overcomplete dictionary matrix consisting of the BS steering vectors corresponding to $N_{1}$ discretized arrival angles. Likewise, $\mathbf{A}_{M S} \in \mathbb{C}^{M \times N_{2}}$ can be obtained using MS steering vectors corresponding to $N_{2}$ discretized departure angles. The matrix $\mathbf{H}_{v} \in \mathbb{C}^{N_{1} \times N_{2}}$ is sparse, with $L$ non-zero entries corresponding to the channel path gains, $\left\{\alpha_{l}\right\}$. By exploiting the Kronecker product property of (4), the received signal can be rewritten as

$$
\begin{aligned}
y(t) & =\mathbf{w}^{H}(t) \mathbf{A}_{B S} \mathbf{H}_{v} \mathbf{A}_{M S}^{H} \mathbf{f}(t) s(t)+n^{\prime}(t) \\
& =\left[\left(\mathbf{A}_{M S}^{H} \mathbf{f}(t)\right)^{T} \otimes\left(\mathbf{w}^{H}(t) \mathbf{A}_{B S}\right)\right] \mathbf{h}+n^{\prime}(t) \\
& =\left(\mathbf{f}^{T}(t) \otimes \mathbf{w}^{H}(t)\right)\left(\mathbf{A}_{M S}^{*} \otimes \mathbf{A}_{B S}\right) \mathbf{h}+n^{\prime}(t)
\end{aligned}
$$


where $\mathbf{h} \triangleq \operatorname{vec}\left(\mathbf{H}_{v}\right)$ and $n^{\prime}(t)$ is the equivalent noise. Collecting the received signals as $\mathbf{y} \triangleq[y(1), \ldots, y(T)]^{T}$, we have

$$
\begin{aligned}
\mathbf{y} & =\left[\begin{array}{c}
\mathbf{f}^{T}(1) \otimes \mathbf{w}^{H}(1) \\
\vdots \\
\mathbf{f}^{T}(T) \otimes \mathbf{w}^{H}(T)
\end{array}\right]\left(\mathbf{A}_{M S}^{*} \otimes \mathbf{A}_{B S}\right) \mathbf{h}+\mathbf{n} \\
& \triangleq \Psi \mathbf{h}+\mathbf{n} .
\end{aligned}
$$

The above model together with the unique characteristics of mmWave time-varying channels can be exploited to estimate AoAs/AoDs. For example, the work in [94] considered the channel estimation problem for multi-user uplink MIMO mmWave communication systems, where both the BS and the users were assumed to have hybrid beamforming structures. The low-rank structure of the received data was exploited within a PARAFAC model and a layered pilot transmission scheme was devised to reduce the training overhead. The conditions to ensure the uniqueness of the decomposition were used for the beamformer design. Similar to [94], the author in [95] considered the problem of downlink channel estimation for mmWave MIMO-OFDM systems. The authors proposed a PARAFAC decomposition-based method for channel parameter estimation, including angles, time delays, and fading coefficients. The analysis revealed that the uniqueness of the CPD could be guaranteed with a small training overhead. The CRLB was also developed as a benchmark for the proposed tensor based algorithm.

The work in [96] combined dual-polarized (DP) antenna arrays with the double directional (DD) channel model for downlink channel estimation. The combination was modeled as a low-rank four-way tensor and tensor decomposition algorithms were used to effectively estimate the associated channel parameters. Furthermore, the DD channel with DP arrays was shown to be identifiable under very mild conditions. In [97], a compressed tensor decomposition algorithm is added to alleviate the training overhead. In [98], the practical hardware impairment, i.e., carrier frequency offset (CFO) was considered. The authors proposed a joint $\mathrm{CFO}$ and channel estimation method based on tensor modeling and compressed sensing which was proved to be more robust to a small number of channel measurements via simulations. The work in [99] discussed the channel estimation problem under a MIMOOFDM transmission assumption. A tensor-based minimum mean square error (MMSE) channel estimator was proposed and then by incorporating a 3D sparse representation into the tensor-based channel model, a tensor compressive sensing (tensor-CS) model is formulated by assuming that the channel is compressively sampled in space (radio-frequency chains), time (symbol periods), and frequency (pilot subcarriers), which is used as the basis for the formulation of a tensororthogonal matching-pursuit (T-OMP) estimator. The work [100] addressed the problem of joint downlink (DL) and uplink (UL) channel estimation for millimeter wave mmWave MIMO systems using a tensor modeling approach. Assuming a closedloop and multifrequency-based channel training framework, the algorithms developed therein jointly estimated both the DL and UL channels by concentrating most of the processing burden for channel estimation at the BS side.

\section{F. BTD for Intersymbol Interference Problem}

Consider the DS-CDMA model in (27). Let $z_{l n}^{m}$ denote the $l$ th chip of the $n$th symbol of the $m$ th user signal. If there is no ISI, then $z_{l n}^{m}=c_{m}(l) s_{m}(n)$. In this case, as in (27), the baseband output for symbol $n$ and chip $l$ from the $k$ th antenna is $x_{k, n, l}=\sum_{m=1}^{M} h_{m}(k) z_{l n}^{m}$. On the other hand, if the ISI exists such that it has an impact over at most $R$ symbols, then we have

$$
z_{l n}^{m}=\sum_{r=0}^{R} \mathbf{E}_{m}(l, r) s_{m}(n-r),
$$

where $\mathbf{E}_{m}(l, r)$ denotes the overall impulse response of the $l$ th chip and the most recent $r$ th symbol. In this case, we can express $x_{k, n, l}$ in tensorial form as

$$
\mathcal{X}=\sum_{m=1}^{M} \mathbf{h}_{m} \circ\left(\mathbf{E}_{m} \mathbf{S}_{m}\right),
$$

where $\mathbf{E}_{m} \in \mathbb{R}^{L \times R}$ and $\mathbf{S}_{m} \in \mathbb{R}^{R \times N}$ is a Toeplitz matrix with $s_{m}(n-r)$ as its $(r, n)$ th element. Eq. (51) admits BTD in rank- $(1, R, R)$ terms. Details of the corresponding essential uniqueness condition and blind deconvolution algorithm can be found in [69], [101].

Recently, coupled tensor decompositions have emerged as an important tool for handling missing data in signal processing and analysis of coupled data sets. The necessary and sufficient uniqueness conditions for coupled decompositions depend on the observed data sampling patterns. For example, the uniqueness conditions and linear algebra based algorithms of coupled CPD and coupled BTD were both considered in [102], [103], where multi-coupled subtensors were formed with partly observed data in the first two dimensions and fully observed data in the third dimension. The coupled tensor decompositions can be summarized as follows.

$$
\begin{aligned}
& \min _{\text {factors }} \text { Loss(subtensors, factors)+penalty(factors) } \\
& \text { subject to constraints(factors) }
\end{aligned}
$$

\section{ApPLICATIONS IN MIMO RADAR}

The higher dimensional signal structures inherent in MIMO radar invite tensor-based signal processing solutions to the target parameter estimation and transmit beamforming problems. The use of tensor models and milti-linear algebraic methods in MIMO radar is not yet at the same level of maturity as in wireless communications, but some interesting results are existing. Below, we give an overview of tensor-based methods in bistatic and monostatic MIMO radar systems.

\section{A. Tensor Techniques for Target Parameter Estimation in MIMO Radar}

For a bistatic MIMO radar with an $M$-element transmit array and an $N$-element receive array, the received signal model under the assumption of orthogonal transmit waveforms and a coherent processing interval (CPI) containing $Q$ pulses can be expressed as [56], [57]

$$
\mathbf{Y}=(\mathbf{A} \diamond \mathbf{B}) \mathbf{C}^{T}+\mathbf{Z},
$$


where $\mathbf{Y} \in \mathbb{C}^{M N \times Q}$ contains the received data after matched filtering, $\mathbf{Z}$ is the spatially and temporally white additive noise, $\mathbf{C}^{T} \in \mathbb{C}^{P \times Q}$ contains the reflection coefficients of $P$ targets corresponding to $Q$ pulses, and $\mathbf{A} \in \mathbf{C}^{M \times P}$ and $\mathbf{B} \in \mathbf{C}^{N \times P}$ denote the respective steering matrices for transmit and receive arrays. Arranging the matched-filter outputs as a tensor $\mathcal{Y} \in \mathbb{C}^{M \times N \times Q}$ and following the definitions of the matrix unfoldings in Section II-B2, it can be observed that model (52) represents the PARAFAC decomposition. As such, target parameter estimation can proceed within the PARAFAC framework.

The target AoAs and AoDs were estimated using the PARAFAC model in [56] under narrowband far-field assumptions. The $p$ th column of matrix A (matrix B) in (52) captures the delays across the different transmit (receive) antennas relative to a reference transmit (receive) antenna for a plane wavefront departing in (arriving from) the direction of the $p$ th target. Two different models for target radar crosssection (RCS) fluctuations were considered. Swerling I model assumes the RCS coefficients to be constant over the CPI, whereas in the Swerling II model, the RCS coefficients vary independently from pulse to pulse. Conditions for essential uniqueness guarantees were established which yielded useful bounds on the number of resolvable targets.

The works in [57], [58], on the other hand, proposed tensorbased near-field localization algorithms for targets located closer to the transmit and/or receive arrays of a bistatic MIMO radar. In this case, the $p$ th column of matrix $\mathbf{A}$ (matrix $\mathbf{B}$ ) was defined in terms of the exact path differences under spherical wavefronts between the reference transmit (receive) antenna and other antennas in the transmit (receive) array for the $p$ th target. The estimated target parameters included the AoAs, the AoDs, and the target distances from transmit and receive reference antennas. In [58], the parameters were obtained through iteratively optimizing a least-squares cost function defined with respect to the elements of $\mathbf{A}$ and $\mathbf{B}$. Alternatively, the authors in [57] first estimated $\mathbf{A}$ and $\mathbf{B}$ as factor matrices in an approximate low rank $\mathrm{CPD}$ of the tensor $\mathcal{Y}$, which were then used to estimate the target parameters by solving systems of linear equations.

We note that the model in (52) as well as the aforementioned methods do not take into account the effects of array mutual coupling (MC) on target parameter estimation. MC however occurs in practice and can lead to performance degradation if not properly compensated. Both higherorder singular value decomposition (HOSVD) and PARAFAC decomposition-based methods have been recently proposed for accurate target localization using MIMO radar in the presence of mutual coupling [59], [104], [105].

A tensor-based sub-Nyquist monostatic MIMO radar was proposed in [106] which used undersampled measurements in spectral, spatial, Doppler, and temporal domains to jointly estimate target AoAs, range, and Doppler. The received signals were modeled as a partial third-order tensor. On-grid target parameters were estimated by solving a sparse recovery problem using tensor orthogonal matching pursuit, whereas a nuclear-norm regularized tensor completion method was employed for off-grid target parameters. The lower bounds on the total numbers of antenna channels, transceiver frequencies, and pulses required for perfect recovery of both on-grid and off-grid targets were also determined.

\section{B. Transmit Array Interpolation in MIMO Radar}

Together with waveform design, transmit beamspace (TB) design [107]-[110] is one of the fundamental problems in MIMO radar with colocated antennas. While designing TB, certain properties such as rotational invariance property (RIP) at the receive array can be ensured via $\mathrm{TB}$ matrix at the transmit array for a monostatic MIMO radar [60]. It is especially useful for reducing significantly the complexity of solving the target localization problem (e.g., AoA estimation - azimuth and elevation for two-dimensional (2-D) arrays) at the receive array. If the RIP is ensured between more than two virtual subarrays (the solution for two subarrays related to each other through RIP is the classical ESPRIT), the received signals in MIMO radar can be arranged in a tensor and tensor algebra then becomes the main tool for designing localization algorithms.

For example, consider a mono-static MIMO radar and assume that the transmit and receive arrays are placed on a plane and have arbitrary geometries. The receive array consists of antenna elements randomly selected from the transmit array. Let the transmit antenna elements be located at the position $\boldsymbol{p}_{m} \triangleq\left[x_{m}, y_{m}\right]^{T}, m=1,2, \ldots, M$. Then the $M \times 1$ steering vector of the transmit array can be expressed as

$$
\boldsymbol{a}(\theta, \phi) \triangleq\left[e^{-j 2 \pi \boldsymbol{u}^{T}(\theta, \phi) \boldsymbol{p}_{1}}, \ldots, e^{-j 2 \pi \boldsymbol{u}^{T}(\theta, \phi) \boldsymbol{p}_{M}}\right]^{T}
$$

where $\boldsymbol{u}(\theta, \phi) \triangleq[\sin \theta \cos \phi, \sin \theta \sin \phi]^{T}$ represents the propagation vector, and $\theta, \phi$ are the elevation and azimuth, respectively. Similarly, the steering vector of the receive array can be then expressed as

$$
\boldsymbol{b}(\theta, \phi) \triangleq\left[e^{-j 2 \pi \boldsymbol{u}^{T}(\theta, \phi) \boldsymbol{p}_{1}}, \ldots, e^{-j 2 \pi \boldsymbol{u}^{T}(\theta, \phi) \boldsymbol{p}_{N}}\right]^{T} .
$$

Let $s_{m}(t)$ be the complex envelope of the $m$ th transmit signal where $t$ represents the fast time, and then $\left\{s_{m}(t)\right\}_{m=1}^{M}$ be a set of $M$ waveforms. Each waveform $s_{m}(t)$ has unit energy, and all waveforms are orthogonal to each other during one pulse, i.e., $\int_{T} s_{m}(t) s_{m^{\prime}}^{*}(t) d t=\delta\left(m-m^{\prime}\right)$, where $T$ is the radar pulse duration, $\delta(\cdot)$ denotes the Dirac delta function, and $L$ is the number of samples per pulse period. The signal radiated towards a spatial region of interest is therefore given by

$$
\zeta(t, \theta, \phi)=\boldsymbol{a}^{T}(\theta, \phi) \boldsymbol{s}(t)=\sum_{m=1}^{M} a_{m}(\theta, \phi) s_{m}(t)
$$

where $s(t) \triangleq\left[s_{1}(t), \ldots, s_{M}(t)\right]^{T}$ and $a_{m}(\theta, \phi)$ is the $m$ th element of $\boldsymbol{a}(\theta, \phi)$.

Assuming that radar cross section (RCS) coefficients obey Swerling II model, for the case of $K$ targets located in a spatial sector of interest, the received MIMO observation vector can be expressed as

$$
\boldsymbol{x}(t, q)=\sum_{k=1}^{K} \beta_{k}(q)\left(\boldsymbol{a}^{T}\left(\theta_{k}, \phi_{k}\right) \boldsymbol{s}(t)\right) \boldsymbol{b}\left(\theta_{k}, \phi_{k}\right)+\boldsymbol{n}(t, q)
$$


where $q$ represent the slow time index, $\beta_{k}(q)$ is the RCS coefficient of $k$ th target with variance $\sigma_{\beta}^{2}$, and $\boldsymbol{n}(t, q)$ is the noise vector modeled as complex spatial and temporal white Gaussian process. Using the orthogonality property of the transmit waveforms, the received data vector corresponding to the $m$ th waveform after matched-filtering can be obtained as

$$
\boldsymbol{y}_{m}(q)=\sum_{k=1}^{K}\left(a_{m}\left(\theta_{k}, \phi_{k}\right) \boldsymbol{b}\left(\theta_{k}, \phi_{k}\right)\right) \beta_{k}(q)+\boldsymbol{z}(q)
$$

where $\boldsymbol{y}_{m}(q) \in \mathbb{C}^{N \times 1}, \boldsymbol{z}(q)$ is the noise vector after matchedfiltering whose covariance matrix is given by $\sigma_{n}^{2} \boldsymbol{I}_{N}$. Hence, the whole receive vector, i.e., the vector that is obtained by stacking $\boldsymbol{y}_{m}(q), m=1, \ldots, M$, one under another, can be written as

$$
\boldsymbol{y}(q)=(\boldsymbol{A}(\theta, \phi) \diamond \boldsymbol{B}(\theta, \phi)) \boldsymbol{\beta}(q)+\boldsymbol{z}(q)
$$

where $\boldsymbol{A}(\theta, \phi) \triangleq\left[\boldsymbol{a}\left(\theta_{1}, \phi_{1}\right), \ldots, \boldsymbol{a}\left(\theta_{K}, \phi_{K}\right)\right]$ is the transmit steering matrix, $\boldsymbol{B}(\theta, \phi) \triangleq\left[\boldsymbol{b}\left(\theta_{1}, \phi_{1}\right), \ldots, \boldsymbol{b}\left(\theta_{K}, \phi_{K}\right)\right]$ is the receive steering matrix, and $\boldsymbol{\beta}(q) \triangleq\left[\beta_{1}(q), \ldots, \beta_{K}(q)\right]^{T}$ is the vector of RCS coefficients during $q$ th pulse.

Using the TB matrix at the transmitter, the received signal in $q$ th pulse is given as

$$
\tilde{\boldsymbol{y}}(q)=\boldsymbol{B}(\theta, \phi) \boldsymbol{\Sigma}(q) \boldsymbol{A}^{H}(\theta, \phi) \boldsymbol{W} \boldsymbol{s}(t)+\boldsymbol{z}(q)
$$

where $\boldsymbol{\Sigma}(q)=\operatorname{diag}(\boldsymbol{\beta}(q))$ and $\boldsymbol{W}$ is the TB matrix. Considering $Q$ pulses, the received 2D TB MIMO radar signal matrix $\tilde{\boldsymbol{Y}} \in \mathbb{C}^{M N \times Q}$ can be formed. Here $\tilde{M}$ is the dimension of the transmit signal after TB transform. Then the corresponding 2D TB MIMO radar tensor model can be expressed as

$$
\mathcal{Y}=\mathcal{A} \times{ }_{R} P+\mathcal{Z}
$$

where steering tensor $\mathcal{A}$ is composed by stacking $K$ targets' steering tensor $\boldsymbol{\mathcal { A }}_{k}$ together, $\boldsymbol{P} \triangleq[\boldsymbol{\beta}(1), \boldsymbol{\beta}(2), \ldots, \boldsymbol{\beta}(Q)]$ contains $K$ vectors of targets' RCS coefficients for $Q$ pulses, and $\mathcal{Z}$ stands of the noise samples, which is assumed to be Gaussian with zero mean, and $R$ represents the $R$ th mode tensor-matrix product.

Note that transmit and receive array geometries are arbitrary here, and do not need to be uniform. Then TB also performs the function of array interpolation as shown in [111]-[116], where the problem of the 2D transmit array interpolation and beamspace design for mono-static MIMO radar with application to elevation and azimuth estimation has been addressed. The 2D transmit array interpolation has been formulated, for example, as the minimax convex optimization problem with constraints on array interpolation errors within a spatial sector of interest while minimizing the transmit power outside the sector. The desired structure of the virtual transmit array (for example, L-shaped array) is then enforced. It allows to benefit from translational invariance property when estimating elevation and azimuth parameter at the receiver. The advantage of the high-dimensional structure inherent in the received signal as explained above (the signal has been folded into a higher-order tensor) allows for the use tensor-based ESPRIT methods.

\section{Tensor Techniques for Parameter Estimation in MIMO} Radar with Arrays of Regular Geometries

The DOA estimation bias caused by transmit array interpolation errors, which are unavoidable for the techniques reviewed in the previous subsection, can be partially compensated by building an offline look-up table aiming to decrease the DOA estimation bias. However, a higher localization accuracy may be achieved if the transmit array also has a regular uniform structure. This is because there will be no loss of accuracy arising due to array interpolation, as shown in [117] where the 2-D transmit array was non-adaptively partitioned into a number of subarrays, each contributing a slice in a data tensor at the receiver. Then TB is adaptively designed for each subarray such that the beampatterns corresponding to each matrix of the TB for each subarray had the exact same magnitude.

For example, in the transmit array is a uniform rectangular array (URA), the design of TB matrix $\mathbf{W}$ can be performed in two stages. First, a TB matrix $\mathbf{U}_{0}=\left[\mathbf{u}_{1}, \ldots, \mathbf{u}_{k}\right]$, with full column rank $K$, is designed over a spatial sector $\Theta=\left[\begin{array}{ll}\theta_{1} & \theta_{2}\end{array}\right]$ and $\Phi=\left[\begin{array}{ll}\phi_{1} & \phi_{2}\end{array}\right]$ using only the first $(P-1)$ rows and $(Q-1)$ columns of the transmit array. Then, a simple transformation is performed on $\mathbf{U}_{0}$ to produce $\mathrm{TB}$ matrices with identical beampatterns, but which correspond to different subarrays as shown in Fig. 7.

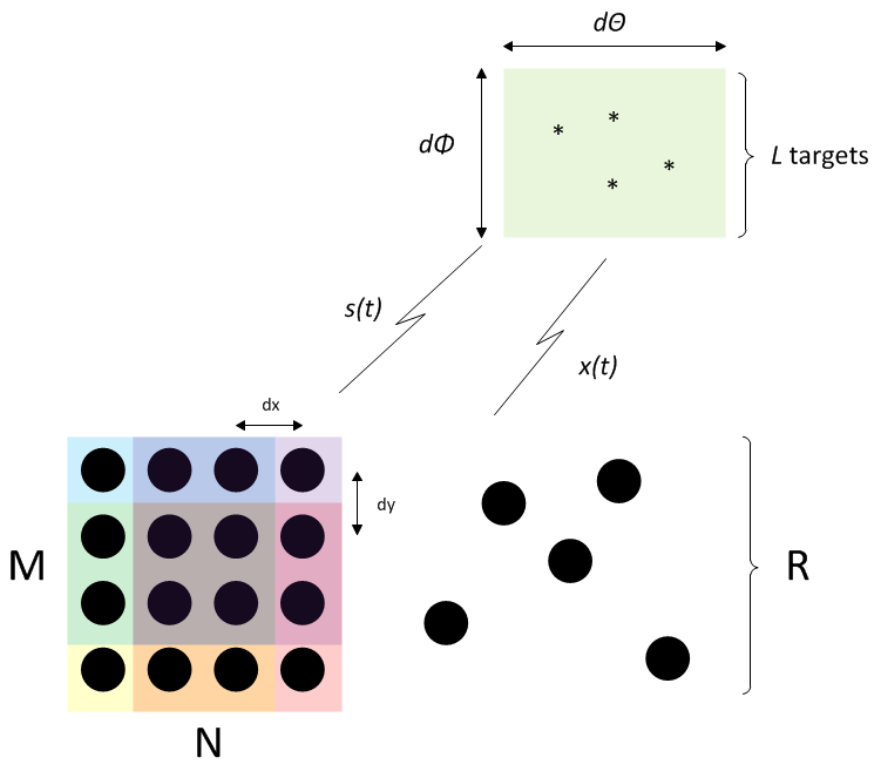

Fig. 7. An example of 2-D uniform rectangular array non-adaptive division to a number of identical subarrays. Different waveforms are sent from different subarrays, but the beampattern shape and RIP between different subarrays is ensured by TB design.

It is trivial to show that a matrix $\mathbf{U}_{0}^{\prime}$ of dimension $P Q \times K$, with an identical beampattern to that of $\mathbf{U}_{0}$, can be constructed by placing zeros in the spots corresponding to the antenna elements which were omitted from the original design of $\mathbf{U}_{0}$. The matrix $\mathbf{U}_{0}^{\prime}$ then denotes a beamforming matrix where $K$ beams are transmitted from the first $P-1$ rows and $Q-1$ columns of a transmit array of dimension $P \times Q$. Given the shape of the transmit array, it is simple to show that by shifting the positions of the zeros in $\mathbf{U}_{0}^{\prime}$, the exact same beampattern 
can be achieved by subarrays containing the first $P-1$ rows and last $Q-1$ columns, the last $P-1$ rows and the first $Q-1$ columns, and finally the last $P-1$ and last $Q-1$ columns of the transmit array. These three matrices are denoted as $\mathbf{U}_{1}^{\prime}$, $\mathbf{U}_{2}^{\prime}$, and $\mathbf{U}_{3}^{\prime}$, respectively. With these matrices defined, it is easy to show that the following is true

$$
\begin{aligned}
\mathbf{a}^{H}(\theta, \phi) \mathbf{U}_{0}^{\prime} & =e^{j 2 \pi d_{x} \sin \theta \cos \phi}\left(\mathbf{a}^{H}(\theta, \phi) \mathbf{U}_{1}^{\prime}\right) \\
& =e^{j 2 \pi d_{y} \sin \theta \sin \phi}\left(\mathbf{a}^{H}(\theta, \phi) \mathbf{U}_{2}^{\prime}\right) \\
& =e^{j 2 \pi\left(d_{x} \sin \theta \cos \phi+d_{y} \sin \theta \sin \phi\right)}\left(\mathbf{a}^{H}(\theta, \phi) \mathbf{U}_{3}^{\prime}\right)
\end{aligned}
$$

The beamforming matrix $\mathbf{W}$ is then defined as $\mathbf{W} \triangleq$ $\left[\mathbf{U}_{0}^{\prime}, \mathbf{U}_{1}^{\prime}, \mathbf{U}_{2}^{\prime}, \mathbf{U}_{3}^{\prime}\right]$ with an overall dimension of $P Q \times 4 K$. Clearly, in the original design problem, the number of resolvable targets $K$ must be no larger than $P Q / 4$.

Given the structure (61) imposed on the beamspace matrix $\mathbf{W}$, let us turn our attention to (59). Rewriting the noiseless matrix before vectorization allows to illustrate the structure of W on DOA estimation. Specifically, we can write that

$$
\mathbf{B}(\theta, \phi) \boldsymbol{\Sigma}(q) \mathbf{A}^{H}(\theta, \phi) \mathbf{W}=\mathbf{B}(\theta, \phi) \boldsymbol{\Gamma}
$$

where $\boldsymbol{\Gamma} \triangleq \boldsymbol{\Sigma}(q) \mathbf{A}^{H}(\theta, \phi) \mathbf{W}$. The matrix $\boldsymbol{\Gamma}$ is the source signal matrix, and has dimension $L \times 4 K$. Let $\boldsymbol{\Gamma}_{0}=\boldsymbol{\Sigma}(\tau) \mathbf{A}^{H} \mathbf{U}_{0}^{\prime}$ is the source signal matrix corresponding to $K$ beams eliminated from the first $(P-1)$ rows and $(Q-1)$ columns of the transmit array. Using the relations (61), we define matrices $\boldsymbol{\Omega}_{i}, i \in\{0,1,2,3\}$ as the $L \times L$ diagonal matrices with the $l$-th diagonal entry of $\Omega_{i}$ being the complex exponential in (61) which relates $\mathbf{a}^{H}(\theta, \phi) \mathbf{U}_{0}^{\prime}$ to $\mathbf{a}^{H}(\theta, \phi) \mathbf{U}_{i}^{\prime}$. The matrix $\boldsymbol{\Omega}_{0}$ is obviously the identity matrix. Then (62) can be expressed as the following block partitioned matrix

$$
\begin{aligned}
\mathbf{B} \boldsymbol{\Gamma} & =\mathbf{B}\left[\boldsymbol{\Omega}_{0} \boldsymbol{\Gamma}_{0}\left|\boldsymbol{\Omega}_{1} \boldsymbol{\Gamma}_{0}\right| \boldsymbol{\Omega}_{2} \boldsymbol{\Gamma}_{0} \mid \boldsymbol{\Omega}_{3} \boldsymbol{\Gamma}_{0}\right] \\
& =\left[\mathbf{B} \boldsymbol{\Omega}_{0}\left|\mathbf{B} \boldsymbol{\Omega}_{1}\right| \cdots \mid \mathbf{B} \boldsymbol{\Omega}_{3}\right] \operatorname{bdiag}_{4}\left(\boldsymbol{\Gamma}_{0}\right)
\end{aligned}
$$

where we drop the dependence of $\mathbf{B}$ on $(\theta, \phi)$ and $\operatorname{bdiag}_{m}(\cdot)$ takes a single matrix as an argument, and creates a block diagonal matrix whose $m$ blocks are equal to its argument. The matrix $\mathbf{B} \boldsymbol{\Omega}_{0}$ is simply the receiver response matrix to $K$ targets. The virtual receiver response matrices $\mathbf{B} \boldsymbol{\Omega}_{1}, \mathbf{B} \boldsymbol{\Omega}_{2}$, and $\mathbf{B} \Omega_{3}$ are exactly the receiver response matrices to $K$ targets, for identical receive arrays that are linearly displaced from our actual receiver by $\left[d_{x}, 0\right],\left[0, d_{y}\right]$, and $\left[d_{x}, d_{y}\right]$, respectively. The source signal matrix $\boldsymbol{\Gamma}_{0}$ is a common factor for each. From (63) it is visible that the structure for $\mathbf{W}$ enforces an algebraic structure on $\mathbf{Y}$ which can be exploited by search-free algorithms for DOA estimation, such as, for example, ESPRIT. Moreover, (63) can be viewed as an unfolding of a tensor with each slice to be one of the 4 matrices $\mathbf{B} \boldsymbol{\Omega}_{i} i=0,1,2,3$.

Matrix $\mathbf{Y}$ has dimension $4 R K \times Q$ and it obtained by unfolding the corresponding tensor $\mathcal{Y}$ of signal for all $Q$ pulses. After defining the matrix selection operator $\mathbf{F}_{i}(\cdot)$ which selects the $(i M / 4+1)-M / 4(i+1)$ rows from an arbitrary matrix with $M$ rows, where $i=0,1,2,3, \mathbf{Y}$ and a new matrix $\mathbf{Y}^{\prime}$ can be expressed as

$$
\mathbf{Y}=\left[\begin{array}{l}
\mathbf{F}_{0}(\mathbf{Y}) \\
\mathbf{F}_{1}(\mathbf{Y}) \\
\mathbf{F}_{2}(\mathbf{Y}) \\
\mathbf{F}_{3}(\mathbf{Y})
\end{array}\right], \quad \mathbf{Y}^{\prime}=\left[\begin{array}{l}
\mathbf{F}_{0}(\mathbf{Y}) \\
\mathbf{F}_{2}(\mathbf{Y}) \\
\mathbf{F}_{1}(\mathbf{Y}) \\
\mathbf{F}_{3}(\mathbf{Y})
\end{array}\right]
$$

Forming the cross correlation matrices $\mathbf{R}_{\mathbf{Y}}=I^{-1} \mathbf{Y} \mathbf{Y}^{H}$ and $\mathbf{R}_{\mathbf{Y}^{\prime}}=I^{-1} \mathbf{Y}^{\prime} \mathbf{Y}^{\prime H}$, and performing ESPRIT on both will yield a vector of $L$ phase arguments which are directly proportional to $\zeta_{l}$ and $\gamma_{l}$. Defining a complex number $z_{l}=\gamma_{l}+j \zeta_{l}$ the angle estimates are given by $\phi_{l}=\arctan \left(\zeta_{l} / \gamma_{l}\right)$, and $\theta_{l}=\left|z_{l}\right|$.

The above described structure of the TB matrix $\mathbf{W}$ is just a special case shown in Fig. 7. However, the approach can be generalized to allow for a flexible subarray selection, allow for more general that just URA transmit array geometries, allow for more computationally efficient tensor decomposition techniques that use the additional structures in the signal tensor. These and other generalizations have been addressed in the recent work [118]. The additional structure in the signal tensor comes from the Vandermonde structure of the factor matrices. The term that was recently coined for decomposition methods of tensors with some additional structures that need to be taken into account and may lead to significant improvement of computational efficiency is constrained factor decomposition [27]. The decomposition methods proposed in [118] belongs then to this class of tensor decomposition techniques.

Finally, an extension of TB design method for AoA/AoD estimation in bistatic MIMO radar has been recently proposed in [119], wherein uniform power distribution across the transmit array elements was achieved via inequality constraints.

\section{Tensor Techniques for Slow-Time MIMO Radar}

A notifiable disadvantage of MIMO radar is the need of multiple orthogonal waveforms. Indeed, multi-waveform MIMO radar demands several signal generators to transmit orthogonal waveforms, which can be costly and unacceptable for especially in such applications as automotive MIMO radar. However, using additionally $M$ phase shifters at the transmit array incited of a multiple orthogonal waveform generator, the implementation of MIMO radar can be still upgraded easily from a legacy SIMO radar, where only one signal generator is used. The associated waveform design approach has been called Doppler division multiple access (DDMA) technique, and the signal model for this techniques can also be represented in tensor form [120]. Such approach is called slow-time MIMO radar, and its essence is to modulate each transmit signal with a unique Doppler shift via a pulse-to-pulse phase coding. Under this circumstance, the model in (52) can be modified to represent the received signal of MIMO radar using DDMA technique as

$$
\mathbf{Y}=\left[(\mathbf{A} \diamond \mathbf{B}) \mathbf{C}^{T}\right] \odot \mathbf{D}+\mathbf{Z}
$$

where $\mathbf{D} \triangleq\left(\mathbf{I}_{M} \diamond \mathbf{1}_{N \times M}\right) \mathbf{P}, \mathbf{P} \in \mathbb{C}^{M \times Q}$ is the phase modulation matrix that achieves waveform diversity in Doppler domain, $\mathbf{1}_{N \times M}$ is a $N \times M$ all-one matrix, and the symbol $\odot$ denotes the Hadamard (element-wise) product. It can be 
observed that matrix $\mathbf{D}$ also represents the parallel factor (PARAFAC) decomposition. It is worth noting that the independence of phase modulation matrix and the received signal can be exploited in the context of slow-time MIMO radar. Based on this property, a novel tensor model was designed to improve the target parameter estimation performance is [120]. Moreover, the additional phases on transmit array lead to a cyclically varying transmit beampattern from pulse to pulse, which can be regarded as a special case of TB design.

\section{CONCLUSION}

In this paper, we presented a comprehensive overview of tensor decompositions in wireless communications and MIMO radar. We provided a description of basic tensor operations and tensor decompositions, thus establishing the preliminaries for advanced application-driven discussions. Within the area of wireless communications, we provided an in-depth description of tensor-based methods for blind symbol recovery and channel parameter estimation, focusing on CDMA, STF, cooperative/relay, MIMO, and mmWave systems. We also reviewed tensor techniques for transmit beamspace design and target parameter estimation in MIMO radar. The presented methods and strategies highlighted the prospects and potential of tensor algebra and decompositions in wireless communications and MIMO radar.

\section{REFERENCES}

[1] A. Cichocki et al. (Mar. 2014). "Tensor decompositions for signal processing applications from two-way to multiway component analysis," IEEE Signal Process Mag., vol. 32, no. 2, pp. 145-163, Mar. 2015, doi: 10.1109/MSP.2013.2297439.

[2] G. Favier and A. A. L. F. de, "Overview of constrained PARAFAC models," EURASIP J. Adv. Signal Process., vol. 2014, no. 1, pp. 4-142, 2014.

[3] N. D. Sidiropoulos, L. De Lathauwer, X. Fu, K. Huang, E. E. Papalexakis and C. Faloutsos, "Tensor Decomposition for Signal Processing and Machine Learning," IEEE Trans. Signal Process., vol. 65, no. 13, pp. 3551-3582, 1 Jul. 2017, doi: 10.1109/TSP.2017.2690524.

[4] Y. Ji, Q. Wang, X. Li and J. Liu, "A Survey on Tensor Techniques and Applications in Machine Learning," IEEE Access, vol. 7, pp. 162950 162990, 2019, doi: 10.1109/ACCESS.2019.2949814.

[5] A. L. F. de Almeida, G. Favier, J. P. C. L. da Costa, and J. C. M. Mota, "Overview of tensor decompositions with applications to communications," in Signals and Images: Advances and Results in Speech, Estimation, Compression, Recognition, Filtering, and Processing, R Coelho, V. Nascimento, R. de Queiroz, J. Romano, and C. Cavalcante, Eds. CRC-Press, Jan. 2016, no. Chapter 12, pp. 325-356.

[6] F. L. Hitchcock, "The expression of a tensor or a polyadic as a sum of products," J. Math. Phys., vol 6, pp. 164-189, 1927.

[7] F. L. Hitchcock, "Multilple invariants and generalized rank of a p-way matrix or tensor," J. Math. Phys., vol 7, pp. 39-79, 1927.

[8] L. R. Tucker, "Implications of factor analysis of three-way matrices for measurement of change," in Problems in Measuring Change, C. W. Harris, Ed., University of Wisconsin Press, 1963, pp. 122-137.

[9] L. R. Tucker, "The extension of factor analysis to three-dimensional matrices," in Contributions to Mathematical Psychology, H. Gulliksen and N. Frederiksen, Eds., Holt, Rinehardt, \& Winston, New York, NY, 1964, pp. 110-127.

[10] L. R. Tucker, "Some mathematical notes on three-mode factor analysis," Psychometrika, vol. 31, no. 3, pp. 279-311, 1966.

[11] J. D. Carroll and J. J. Chang, "Analysis of individual differences in multidimensional scaling via an N-way generalization of "EckartYoung" decomposition," Psychometrika, vol. 35, no. 3, pp. 283-319, 1970.

[12] R. A. Harshman, "Foundations of the PARAFAC procedure: Models and conditions for an "explanatory" multi-modal factor analysis," UCLA Working Papers in Phonetics, vol. 16, pp. 1-84, 1970.
[13] A. Smilde, R. Bro, and P. Geladi, Multi-Way Analysis: Applications in the Chemical Sciences, Wiley, Hoboken, NJ, 2004.

[14] P. Kroonenberg, Applied Multiway Data Analysis, Wiley, Hoboken, NJ, 2008.

[15] L. De Lathauwer, J. Castaing, and J.-F. Cardoso, "Fourth-order cumulant based blind identification of underdetermined mixtures," IEEE Trans. Signal Process., vol. 55, no. 6, pp. 2965-2973, 2007.

[16] L. De Lathauwer and A. de Baynast, "Blind deconvolution of DS-CDMA signals by means of decomposition in rank-( $1, L, L)$ terms," IEEE Trans. Signal Process., vol. 56, no. 4, pp. 1562-1571, 2008.

[17] L. De Lathauwer and B. De Moor, "From matrix to tensor: Multilinear algebra and signal processing," in Mathematics in Signal Processing IV, J. McWhirter and I. Proudler, Eds., Clarendon Press, Oxford, UK, 1998, pp. $1-15$.

[18] C. A. R. Fernandes, J. Du, A. P. da Silva, and A. L. F. de Almeida, "Applications of tensor models in wireless communications and mobile computing", Wirel. Commun. Mob. Comput., pp. 7393080:1-393080:2, 2020. doi: $10.1155 / 2020 / 7393080$.

[19] A. J. Paulraj, D. A. Gore, R. U. Nabar, and H. Bolcskei, "An overview of MIMO communications: A key to gigabit wireless," Proc. IEEE, vol. 92, no. 2, pp. 198-218, Feb. 2004.

[20] B. Hochwald, T. L. Marzetta, and C. B. Papadias, "A transmitter diversity scheme for wideband CDMA systems based on space-time spreading," IEEE J. Sel. Areas Commun., vol. 19, no. 1, pp. 48-60, 2001.

[21] H. Huang, H. Viswanathan, and G. J. Foschini, "Multiple antennas in cellular CDMA systems: transmission, detection, and spectral efficiency," IEEE Trans. Wireless Commun., vol. 1, no. 3, pp. 383-392, 2002.

[22] R. Doostnejad, T. J. Lim, and E. Sousa, "Space-time spreading codes for a multiuser MIMO system," in Proc. 36th Asilomar Conf. Signals, Syst. Comp., Pacific Grove, CA, Nov. 2002, pp. 1374-1378.

[23] N. D. Sidiropoulos,, G. B. Giannakis, and R. Bro, "Blind PARAFAC receivers for DS-CDMA systems," IEEE Trans. Signal Process., vol. 48, no. 3, pp. 810-823, 2000.

[24] Y. Rong, S.A. Vorobyov, A.B. Gershman, and N.D. Sidiropoulos, "Blind spatial signature estimation via time-varying user power loading and parallel factor analysis," IEEE Trans. Signal Process., vol. 53, no. 5, pp. 1697-1710, 2005.

[25] A. L. F. de Almeida, G. Favier, and J. C. M. Mota, "PARAFACbased unified tensor modeling for wireless communication systems with application to blind multiuser equalization," Signal Process., vol. 87, no. 2, pp. 337-351, 2007.

[26] N. D. Sidiropoulos and G. Z. Dimic, "Blind multiuser detection in WCDMA systems with large delay spread," IEEE Signal Process. Lett., vol. 8, no. 3, pp. 87-89, 2001.

[27] A. L. F. de Almeida, G. Favier, and J. C. M. Mota, "A constrained factor decomposition with application to MIMO antenna systems," IEEE Trans. Signal Process., vol. 56, no. 6, pp. 2429-2442, Jun. 2008, doi: 10.1109/TSP.2008.917026.

[28] A. L. F. de Almeida, G. Favier, and J. C. M. Mota, "Constrained tensor modeling approach to blind multiple-antenna CDMA schemes," IEEE Trans. Signal Process., vol. 56, no. 6, pp. 2417-2428, 2008.

[29] G. R. Stuber, J. R. Barry, S. W Mclaughlin, Y. Li, M. A. Ingram, and T. G. Pratt, "Broadband MIMO-OFDM wireless communications," Proc. IEEE, vol. 92, no. 2, pp. 271-294, 2004.

[30] D. Agrawal, V. Tarokh, A. Naguib, and N. Seshadri, "Space-time coded OFDM for high data-rate wireless communications over wideband channels," in Proc. Veh. Technol. Conf., Ottawa, Canada, 1998, pp. 22322236.

[31] H. Bolcskei and A. Paulraj, "Space-frequency coded broadband OFDM systems," in Proc. Wireless Commun. Netw. Conf., Chicago, IL, 2000, pp. 1-6.

[32] W. Su, Z. Safar, and K. J. R. Liu, "Full-rate full-diversity spacefrequency codes with optimum coding advantage," IEEE Trans. Inf. Theory, vol. 51, no. 1, pp. 229-249, 2005.

[33] W. Su, Z. Safar, and K. J. R. Liu, "Towards maximum achievable diversity in space, time and frequency: Performance analysis and code design," IEEE Trans. Commun., vol. 4, no. 4, pp. 847-1857, 2005.

[34] N. D. Sidiropoulos and R. S. Budampati, "Khatri-Rao space-time codes," IEEE Trans. Signal Process., vol. 50, no. 10, pp. 2396-2407, 2002.

[35] A. L. F. de Almeida, G. Favier, "Unified tensor model for spacefrequency spreading-multiplexing (SFSM) MIMO communication systems," EURASIP J. Adv. Signal Process, vol. 2013, pp. 48, 2013.

[36] G. Favier, M. N. Costa, A. L. F. de Almeida, and J. M. T. Romano, "Tensor space-time (TST) coding for MIMO wireless communication systems," Signal Process., vol. 92, no. 4, pp. 1079-1092, 2012. 
[37] A. L. F. de Almeida, G. Favier, and J. C. M. Mota, "Space-time spreading-multiplexing for MIMO wireless communications systems using the PARATUCK-2 tensor model," Signal Process., vol. 89, no. 11 , pp. 2103-2116, 2009.

[38] A. L. F. de Almeida, G. Favier, and L. R. Ximenes, "Space-timefrequency (STF) MIMO communication systems with blind receiver based on a generalized PARATUCK2 model," IEEE Trans. Signal Process., vol. 61, no. 8, pp. 1895-1909, 2013.

[39] G. Favier and A. L. F. de Almeida, "Tensor space-time-frequency coding with semi-blind receivers for MIMO wireless communication systems," IEEE Trans. Signal Process., vol. 62, no. 22, pp. 5987-6002, 2014.

[40] G. T. de Araújo and A. L. F. de Almeida, "Closed-form channel estimation for MIMO space-time coded systems using a fourth-order tensor-based receiver," Circuits Syst. Signal Process., vol. 37, no. 3, pp. 1343-1357, 2017.

[41] X. Han, A. L. F. de Almeida, and Z. Yang, "Channel estimation for MIMO multi-relay systems using a tensor approach," EURASIP J. Advances in Signal Process., vol. 163, no. 1, 2014.

[42] Y. Rong, M. R. A. Khandaker, and Y. Xiang, "Channel estimation of dual-hop MIMO. relay system via parallel factor analysis," IEEE Trans. Wireless Commun., vol. 11, no. 6, pp. 2224-2233, 2012.

[43] L. R. Ximenes, G. Favier, and A. L. F. de Almeida, "Semi-blind receivers for non-regenerative cooperative MIMO communications based on nested PARAFAC modeling," IEEE Trans. Signal Process., vol. 63, no. 18, pp. 4985-4998, 2015.

[44] J. Li and P. Stoica (Ed.), MIMO Radar Signal Processing, John Wiley \& Sons, Hoboken, NJ, 2009

[45] J. Bergin and J. R. Guerci, MIMO Radar: Theory and Application, Artech House, Boston, MA, 2018.

[46] K. W. Forsythe and D. W. Bliss, "MIMO radar waveform constraints for GMTI," IEEE J. Sel. Topics Signal Process., vol. 4, no. 1, pp. 21-32, Feb. 2010

[47] P. Lombardo, D. Pastina and F. Turin, "Ground moving target detection based on MIMO SAR systems," IEEE J. Sel. Topics Appl. Earth Observ. Remote Sens., vol. 8, no. 11, pp. 5081-5095, 2015.

[48] G. J. Frazer, "Experimental results for MIMO methods applied in overthe-horizon radar," IEEE Aerosp. Electronic Syst. Mag., vol. 32, no. 12, pp. 52-69, 2017.

[49] J. Hu, M. Li, Q. He, Z. He and R. S. Blum, "Joint Estimation of MIMOOTH Radar Measurements and Ionospheric Parameters," IEEE Trans. Aerosp. Electronic Syst., vol. 53, no. 6, pp. 2789-2805, 2017.

[50] Y. I. Abramovich, G. J. Frazer and B. A. Johnson, "Principles of modeselective MIMO OTHR," IEEE Trans. Aerosp. Electronic Syst., vol. 49, no. 3, pp. 1839-1868, 2013.

[51] S. Sun, A. P. Petropulu and H. V. Poor, "MIMO radar for advanced driver-assistance systems and autonomous driving: Advantages and challenges," IEEE Signal Process. Mag., vol. 37, no. 4, pp. 98-117, 2020.

[52] S. Saponara, M. S. Greco and F. Gini, "Radar-on-chip/in-package in autonomous driving vehicles and intelligent transport systems: Opportunities and challenges," IEEE Signal Process. Mag., vol. 36, no. 5, pp. 71-84, 2019.

[53] F. Liu, C. Masouros, A. P. Petropulu, H. Griffiths and L. Hanzo, "Joint radar and communication design: Applications, state-of-the-art, and the road ahead," IEEE Trans. Commun., vol. 68, no. 6, pp. 3834-3862, 2020.

[54] A. Hassanien, M. G. Amin, E. Aboutanios and B. Himed, "Dual-function radar communication systems: A solution to the spectrum congestion problem," IEEE Signal Process. Mag., vol. 36, no. 5, pp. 115-126, 2019.

[55] A. Hassanien, M. G. Amin, Y. D. Zhang and F. Ahmad, "Signaling strategies for dual-function radar communications: an overview," IEEE Aerosp. Electronic Syst. Mag., vol. 31, no. 10, pp. 36-45, 2016.

[56] D. Nion and N. D. Sidiropoulos, "Tensor algebra and multidimensional harmonic retrieval in signal processing for MIMO radar," IEEE Trans. Signal Process., vol. 58, no. 11, pp. 5693-5705, 2010

[57] I. Podkurkov and L. Hamidullina and E. Traikov and M. Haardt and A. Nadeev, "Tensor-based near-field localization in bistatic MIMO radar systems," in Proc. 22nd Int. ITG Workshop on Smart Antennas, Bochum, Germany, 2018, pp. 1-8.

[58] P. R. Singh and Y. Wang and P. Chargé,"Bistatic MIMO radar for near field source localisation using PARAFAC," Electronics Letters, vol. 52, no. 12 , pp. $1060-1061,2016$.

[59] X. Wang and W. Wang and J. Liu and Q. Liu and B. Wang, "Tensorbased real-valued subspace approach for angle estimation in bistatic MIMO radar with unknown mutual coupling," Signal Process., vol. 116, pp. $152-158,2015$.
[60] A. Khabbazibasmenj and A. Hassanien and S.A. Vorobyov and M. W. Morency, "Efficient transmit beamspace design for search-free based DOA estimation in MIMO radar," IEEE Trans. Signal Process., vol. 62, no. 6, pp. 1490-1500, 2014.

[61] T.G. Kolda and B.W. Bader, "Tensor decompositions and applications," Siam Review, vol. 51, no. 3, pp. 455-500, 2009.

[62] P. Comon, "Tensors : A brief introduction," IEEE Signal Process. Mag., vol. 31, no. 3, pp. 44-53, 2014.

[63] L. Grasedyck, D. Kressner, and C. Tobler, "A literature survey of low rank tensor approximation techniques," GAMM-Mitteilungen, vol. 36, no. 1, pp. 53-78, 2013.

[64] A. L. F. de Almeida. "Tensor modeling and signal processing for wireless communication systems," Networking and Internet Architecture, Université de Nice Sophia Antipolis, 2007.

[65] L. De Lathauwer, "Decompositions of a higher-order tensor in block terms - Part II: Definitions and uniqueness," SIAM J. Matrix Analysis and Applications, vol. 30, no. 3, pp. 1033-1066, 2008.

[66] L. De Lathauwer and D. Nion, "Decompositions of a higher-order tensor in block terms - Part III: Alternating least squares algorithms," SIAM J. Matrix Analysis and Applications, vol. 30, no. 3, pp. 1067-1083, 2008.

[67] L. Sorber, M. Van Barel, and L. De Lathauwer, "Optimization-based algorithms for tensor decompositions: Canonical polyadic decomposition, decomposition in rank-(1_r,1_r,1) terms, and a new generalization," SIAM J. Optimization, vol. 23, no. 2, pp. 695-720, 2013.

[68] L. De Lathauwer, "Blind separation of exponential polynomials and the decomposition of a tensor in rank-(1_r,1_r,1) terms," SIAM J. Matrix Analysis and Applications, vol. 32, no. 4, pp. 1451-1474, 2011.

[69] L. De Lathauwer, "Block component analysis, a new concept for blind source separation," in Int. Conf. Latent Variable Analysis and Signal Separation, Springer, 2012, pp. 1-8.

[70] S. A Vorobyov, Y. Rong, N. D. Sidiropoulos, A. B. Gershman, "Robust iterative fitting of multilinear models," IEEE Trans. Signal Processing, vol. 53, no. 8, pp. 2678-2689, 2005.

[71] E. E. Papalexakis, C. Faloutsos, N. D. Sidiropoulos, "Parcube: Sparse parallelizable tensor decompositions," in Proc. Joint European Conference on Machine Learning and Knowledge Discovery in Databases, Sept. 2012, pp. 521-536.

[72] A. P. Liavas, N. D. Sidiropoulos, "Parallel algorithms for constrained tensor factorization via alternating direction method of multipliers," IEEE Trans. Signal Processing, vol. 63, no. 20, pp. 5450-5463, 2015.

[73] M. Sørensen and L. De Lathauwer, "Coupled tensor decompositions for applications in array signal processing," in Proc. IEEE 5th Int. Workshop Computational Advances in Multi-Sensor Adaptive Process., 2013, pp. 228-231.

[74] G. Zhang, X. Fu, J. Wang, X.-L. Zhao, and M. Hong, "Spectrum cartography via coupled block-term tensor decomposition," IEEE Trans. Signal Process., vol. 68, pp. 3660-3675, 2020.

[75] G. Zhang, X. Fu, K. Huang, and J. Wang, "Hyperspectral superresolution: A coupled nonnegative block-term tensor decomposition approach," in Proc. IEEE 8th Int. Workshop Computational Advances in Multi-Sensor Adaptive Process., 2019, pp. 470-474.

[76] Y. Qi, P. Comon, and L. H. Lim, "Uniqueness of nonnegative tensor approximations," IEEE Trans. Inf. Theory, vol. 62, no. 4, pp. 21702183, 2016.

[77] N. D. Sidiropoulos and R. Bro. "On the uniqueness of multilinear decomposition of N-way arrays," J. Chemometrics, vol. 14, pp. 229$239,2000$.

[78] L. Chiantini and G. Ottaviani, "On Generic Identifiability of 3-Tensors of Small Rank," SIAM J. Matrix Anal. Appl., vol. 33, no. 3, pp. 10181037, 2012.

[79] Harshman, R.A., Lundy, M.E. "Uniqueness proof for a family of models sharing features of Tucker's three-mode factor analysis and PARAFAC/candecomp." Psychometrika, vol, 61, pp. 133-154, 1996.

[80] G. Favier, A.L.F. de Almeida, "Overview of constrained PARAFAC models," EURASIP J. Adv. Signal Process. 2014, 142 (2014).

[81] J. B. Kruskal, "Three-way arrays: Rank and uniqueness or trilinear decompositions, with applications to arithmetic complexity and statistics," Linear Algebra Appl., vol. 18, pp. 95-138, 1977.

[82] C. A. R. Fernandes, A. L. F. de Almeida, and D. B. da Costa, "Unified tensor modeling for blind receivers in multiuser uplink cooperative systems," IEEE Signal Process. Lett., vol. 19, no. 5, pp. 247-250, 2012.

[83] L. R. Ximenes, G. Favier, A. L. F. de Almeida, and Y. C. B. Silva, "PARAFAC-PARATUCK semi-blind receivers for two-hop cooperative MIMO relay systems," IEEE Trans. Signal Process., vol. 62, no. 14, pp. 3604-3615, 2014. 
[84] G. Favier, C. A. Fernandes, and A. L. F. de Almeida, "Nested Tucker tensor decomposition with application to MIMO relay systems using tensor space-time coding (TSTC)," Signal Process., vol. 128, pp. 318$331,2016$.

[85] Í. V. Cavalcante, A. L. F. de Almeida, and M. Haardt, "Joint channel estimation for three-hop MIMO relaying systems," IEEE Signal Process. Lett., vol. 22, no. 12, pp. 2430-2434, 2015.

[86] W. C. Freitas, G. Favier, G., and A. L. F. de Almeida, "Sequential closed-form semi-blind receiver for space-time coded multi-hop relaying systems," IEEE Signal Process. Lett., vol. 24, no. 12, pp. 1773-1777, 2017.

[87] Z. Wang, J. Wang, C. Xing, Z. Fei, and J. Kuang, "Tensor-based blind signal recovery for multi-carrier amplify-and-forward relay networks," Science China Information Sciences, vol. 57, no. 10, pp. 1-11, 2014.

[88] W. Freitas, G. Favier, A. L. F. de Almeida, "Generalized Khatri-Rao and Kronecker space-time coding for MIMO relay systems with closed-form semi-blind receivers," Signal Process., vol.151, pp. 19-31, 2018.

[89] B. Sokal, A. L. F. de Almeida, M. Haardt, "Semi-blind receivers for MIMO multi-relaying systems via rank-one tensor approximations," Signal Process., vol. 166, pp. 107-254, 2020.

[90] D. Nion and N. D. Sidiropoulos "Adaptive algorithms to track the PARAFAC decomposition of a third-order tensor," IEEE Trans. Signal Process, vol. 57, no. 6, pp. 2299-2310, 2009.

[91] L. Cheng, G. Yue, D. Yu, Y. Liang, and S. Li, "Millimeter wave timevarying channel estimation via exploiting block-sparse and low-rank structures," IEEE Access, vol. 7, pp. 123355-123366, 2019.

[92] Q. Qin, G. Lin, P. Cheng, and B. Gong, "Time-varying channel estimation for millimeter wave multiuser MIMO systems," IEEE Trans. Veh. Technol., vol. 67, no. 10, pp. 9435-9448, 2018.

[93] L. Cheng, G. Yue, X. Xiong, Y. Liang and S. Li, "Tensor decompositionaided time-varying channel estimation for millimeter wave MIMO systems," IEEE Wireless Commun. Lett., vol. 8, no. 4, pp. 1216-1219, 2019.

[94] Z. Zhou, J. Fang, L. Yang, H. Li, Z. Chen, and S. Li, "Channel estimation for millimeter-wave multiuser MIMO systems via PARAFAC decomposition," IEEE Trans. Wireless Commun., vol. 15, no. 11, pp. 7501-7516, 2016.

[95] Z. Zhou, J. Fang, L. Yang, H. Li, Z. Chen and R. S. Blum, "Lowrank tensor decomposition-aided channel estimation for millimeter wave MIMO-OFDM systems," IEEE J. Sel. Areas Commun., vol. 35, no. 7, pp. 1524-1538, 2017.

[96] C. Qian, X. Fu, N. D. Sidiropoulos, and Y. Yang, "Tensor based parameter estimation of double directional massive MIMO channel with dual-polarized antennas," in Proc. IEEE Int. Conf. Acoust., Speech, Signal Process., Calgary, Canada, 2018, pp. 3884-3888.

[97] C. Qian, X. Fu, N. D. Sidiropoulos, and Y. Yang, "Tensor-based channel estimation for dual-polarized massive MIMO systems," IEEE Trans. Signal Process., vol. 66, no. 24, pp. 6390-6403, 2018.

[98] L. N. Ribeiro, A. L. F. de Almeida, N. J. Myers and R. W. Heath, "Tensor-based Estimation of mmWave MIMO Channels with Carrie Frequency Offset," in Proc. ICASSP 2019, Brighton, United Kingdom, 2019, pp. 4155-4159.

[99] D. C. Araújo, A. L. F. de Almeida, J. P. C. L. Da Costa and R. T. de Sousa, "Tensor-Based Channel Estimation for Massive MIMO-OFDM Systems," IEEE Access, vol. 7, pp. 42133-42147, 2019.

[100] P. R. B. Gomes, A.L. F. de Almeida, J. P. C. L. da Costa, R. T. de Sousa "Joint DL and UL Channel Estimation for Millimeter Wave MIMO Systems Using Tensor Modeling", Wirel. Commun. Mob. Comput., vol. 2019, 2019.

[101] L. De Lathauwer and A. de Baynast, "Blind deconvolution of DSCDMA signals by means of decomposition in rank- $(1,1,1)$ terms," IEEE Trans. Signal Process., vol. 56, no. 4, pp. 1562-1571, 2008.

[102] M. Sørensen and L. D. De Lathauwer, "Coupled canonical polyadic decompositions and (coupled) decompositions in multilinear rank-

[105] F. Wen and Z. Zhang and K. Wang and G. Sheng and G. Zhang, "Angle estimation and mutual coupling self-calibration for ULA-based bistatic MIMO radar," Signal Process., vol. 144, no. Supplement C, pp. 61-67, 2017. (1_r,n,1_r,n,1) terms - Part I: Uniqueness," SIAM J. Matrix Analysis and Applications, vol. 36, no. 2, pp. 496-522, 2015.

[103] M. Sørensen, I. Domanov, and L. De Lathauwer, "Coupled canonical polyadic decompositions and (coupled) decompositions in multilinear rank-(1_r,n,_rn,n,1) terms - Part II: Algorithms," SIAM J. Matrix Analysis and Applications, vol. 36, no. 3, pp. 1015-1045, 2015.

[104] F. Wen and X. Xiong and Z. Zhang, "Angle and mutual coupling estimation in bistatic MIMO radar based on PARAFAC decomposition," Digital Signal Process., vol. 65, pp. 1-10, 2017.

[106] S. Na, K. V. Mishra, Y. Liu, Y. C. Eldar and X. Wang, "TenDSuR: Tensor-Based 4D Sub-Nyquist Radar," in IEEE Signal Processing Letters, vol. 26, no. 2, pp. 237-241, Feb. 2019, doi: 10.1109/LSP.2018.2885617.

[107] A. Hassanien and S. A. Vorobyov, "Phased-MIMO radar: A tradeoff between phased-array and MIMO radars," IEEE Trans. Signal Process. vol. 58, no. 6, pp. 3137-3151, Jun. 2010.

[108] A. Hassanien and S. A. Vorobyov, "Transmit energy focusing for DOA estimation in MIMO radar with colocated antennas," IEEE Trans. Signal Process., vol. 59, no. 6, pp. 2669-2682, Jun. 2011.

[109] A. Khabbazibasmenj, S. A. Vorobyov, A. Hassanien, and M. W. Morency, "Transmit beamspace design for direction finding in colocated MIMO radar with arbitrary receive array and even number of waveforms," in Proc. 46th Annual Asilomar Conf. Signals, Systems, and Computers, Pacific Grove, California, USA, pp. 1307-1311, Nov. 4-7, 2012.

[110] A. Khabbazibasmenj, A. Hassanien, S.A. Vorobyov, and M. W. Morency, "Efficient transmit beamspace design for search-free based DOA estimation in MIMO radar," IEEE Trans. Signal Process., vol. 62 , no. 6, pp. 1490-1500, Mar. 2014

[111] A. Hassanien, S. A. Vorobyov, and J.-Y. Park, "Joint transmit array interpolation and transmit beamforming for source localization in MIMO radar with arbitrary arrays," in Proc. 38th IEEE ICASSP, Vancouver, BC, Canada, May 2013, pp. 4139-4143.

[112] A. Hassanien, S. A. Vorobyov, Y.-S. Yoon, and J.-Y. Park, "RootMUSIC based source localization using transmit array interpolation in MIMO radar with arbitrary planar array," in Proc. IEEE CAMSAP 2013, The Friendly Island, Saint Martin, Dec. 2013, pp. 396-399.

[113] A. Hassanien, M. W. Morency, A. Khabbazibasmenj, S.A. Vorobyov, J.-Y. Park, and S.-J. Kim, "Two-dimensional transmit beamforming for MIMO radar with sparse symmetric arrays," in Proc. IEEE Radar Conf., Ottawa, ON, Canada, Apr. 29-May 3, 2013, pp. 1-6.

[114] A. Hassanien, S. A. Vorobyov, and A. Khabbazibasmenj, "Transmit radiation pattern invariance in MIMO radar with application to DOA estimation," IEEE Signal Process. Letts., vol. 22, no. 10, pp. 1609-1613, Oct. 2015

[115] M.-Y. Cao, S. A. Vorobyov and X. Mao, "Elevation and azimuth estimation in arbitrary planar mono-static MIMO radar via tensor decomposition," in Proc. 2016 IEEE Statistical Signal Processing Workshop (SSP), Palma de Mallorca, Spain, pp. 243-247, Jun. 2016.

[116] M.-Y. Cao and S.A. Vorobyov and A. Hassanien, "Transmit array interpolation for DOA estimation via tensor decomposition in 2D MIMO radar," IEEE Trans. Signal Process., vol. 65, no. 19, pp. 522-5239, 2017.

[117] M.W. Morency and S.A. Vorobyov, "Partially adaptive transmit beamforming for search free 2D DOA estimation in MIMO radar," in Proc. 23rd European Signal Process. Conf., Nice, France, Aug. 31-Sept. 4, 2015, pp. 2676-2680.

[118] F. Xu, M. W. Morency, and S. A. Vorobyov, "DOA estimation for transmit beamspace MIMO radar via tensor decomposition with Vandermonde factor matrix," http://arxiv.org/abs/2101.12666, Jan. 29, 2021.

[119] Z. Huang and B. Tang and H. Wang and L. Qin,"Transmit beamspace design for bistatic MIMO radar with improved angle estimation," IEEE Access, vol. 7, pp. 106204-106212, 2019.

[120] F. Xu, S. A. Vorobyov, and X. Yang, "Joint DOD and DOA estimation in slow-time MIMO radar via PARAFAC decomposition," IEEE Signal Processing Letters, vol. 27, pp. 1495-1499, 2020. (also extended version https://arxiv.org/abs/2007.15738) 